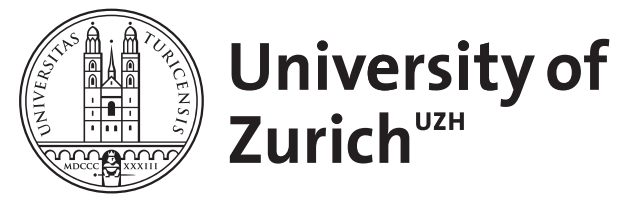

\title{
Families of toric varieties
}

Halic, M

\begin{abstract}
The goal of this article is to construct families of complete toric varieties over arbitrary bases, and to compute the cohomology of the total space. (ㅇ 2003 WILEY-VCH Verlag GmbH Co. KGaA, Weinheim)
\end{abstract}

DOI: https://doi.org/10.1002/mana.200310113

Posted at the Zurich Open Repository and Archive, University of Zurich ZORA URL: https://doi.org/10.5167/uzh-21877

Journal Article

Accepted Version

Originally published at:

Halic, M (2003). Families of toric varieties. Mathematische Nachrichten, 261/26:60-84.

DOI: https://doi.org/10.1002/mana.200310113 


\title{
FAMILIES OF TORIC VARIETIES
}

\author{
MIHAI HALIC
}

The purpose of this note is to present a construction for families of complete toric varieties, which extends the standard one of a single variety. The construction we are going to describe is very much in the spirit of [1], where toric varieties are described as quotients of open subsets in affine spaces: here we simply replace the affine space by the total space of a vector bundle over an arbitrary base variety.

Such families naturally occur in the study of moduli spaces of curves on toric varieties (see [5]) or, alternatively, as the space of solutions of certain vortex-type equations on a Riemann surface (see [9]). Integration on these moduli spaces do furnish invariants, in this case for the toric variety we start with. It is therefore important to be able to perform explicit computations in cohomology, and this in turn requires a good description of the cohomology ring of the moduli space.

With this motivation in mind, we will construct and will study families of toric varieties for their own sake. The structure of the paper is a very linear one: as usual, the first section is devoted to set up the stage. We review the construction of toric varieties as quotients of open subsets in affine spaces, but it is our intension to emphasize the coordinate free features of it. The coordinate free treatement will eventually enable us to extend the construction to the relative setting.

This is done in the second section: we prove that is possible to 'paste' complete toric varieties together, and to obtain a relative version of the standard construction of toric varieties. The objects we get in this way are categorical quotients of Zariski open subsets in the total space of a vector bundle over an arbitrary base, and they are natural generalizations of the projectivized bundle of a vector bundle.

The next step is to compute the cohomology ring of families of complete and simplicial toric varieties. We make use of the computation in [2] of the cohomology ring of complete and simplicial toric varieties and we find that the structure of the cohomology ring in the relative setting is similar to the standard case.

We conclude the article with an overwiew of the situation appearing in $[5,9]$ in the context of the gauged sigma models for toric varieties, which was our primary motivation for this study, and we place it into the more formal frame of the present paper.

Acknowledgements I wish to thank prof. Ch. Okonek for encouraging me to develop this subject, and for his critical remarks.

\section{SOME PRELIMINARY RESULTS}

As we have already said in the introduction, we start with a remark concerning the primitive families of complete fans. Let $\Sigma \subset N_{\mathbb{R}}$, with $N \cong \mathbb{Z}^{n}$, be a complete fan and denote by $\Xi$ the set of generators of the 1-dimensional cones of $\Sigma$ : for each $\xi \in \Xi, \mathbb{R}_{\geq 0} \xi \in \Sigma(1)$ and $\xi$ is the generator of the semi-group $\mathbb{R}_{\geq 0} \xi \cap N$; moreover, we denote by $\left(f_{\xi}\right)_{\xi \in \Xi}$ the canonical basis of $\mathbb{Z}^{\Xi}$. Putting $M:=N^{\vee}$, we obtain the standard short exact sequence 


$$
\begin{aligned}
0 \longrightarrow M & \stackrel{a}{\longrightarrow} \mathbb{Z}^{\Xi} \stackrel{c}{\longrightarrow} A_{\Xi} \longrightarrow 0, \quad \text { where } A_{\Xi}:=\operatorname{Coker}(a), \\
m & \longmapsto \sum_{\xi \in \Xi}\langle m, \xi\rangle f_{\xi} .
\end{aligned}
$$

Definition 1.1. We say that $\xi^{\prime}, \xi^{\prime \prime} \in \Xi$ are equivalent, and we write $\xi^{\prime} \sim \xi^{\prime \prime}$, if $c\left(f_{\xi^{\prime}}\right)=c\left(f_{\xi^{\prime \prime}}\right)$.

Our first goal in this section is to prove the

Proposition 1.2. Let $\Sigma$ be a complete fan and consider $\xi^{\prime}, \xi^{\prime \prime} \in \Xi$. If $\pi \subset \Xi$ is a primitive family, $\xi^{\prime} \in \pi$ and $\xi^{\prime} \sim \xi^{\prime \prime}$, then $\xi^{\prime \prime} \in \pi$ too.

Before starting the proof, let us notice that in the proposition the restriction on $\Sigma$ to be complete is essential. Consider for instance the regular fan

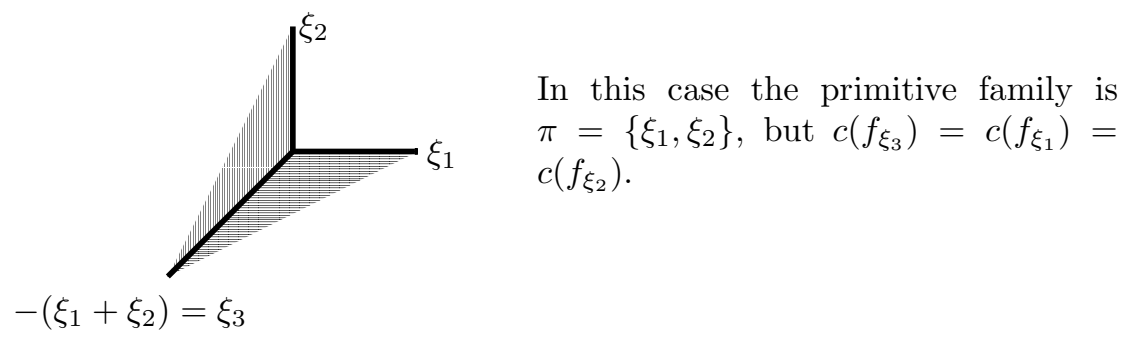

Proof. Let $R=\mathbb{C}\left[Z_{\rho} ; \rho \in \Xi\right]$ be the homogeneous coordinate ring of the toric variety $X_{\Sigma}$ defined by $\Sigma$. The group $G_{\Xi}:=\operatorname{Hom}_{\mathbb{Z}}\left(A_{\Xi}, \mathbb{C}^{*}\right)$ acts on $R$ and the weights of the action define a graduation of $R$ (see [1], section 1). Let us denote by

$$
R=\bigoplus_{\beta \in A \equiv} R_{\beta}
$$

the graded pieces of $R$, and by $\operatorname{Aut}_{g}(R)$ the group of graded automorphisms of $R$. We know by [1], theorem 2.1, that $X_{\Sigma}$ is canonically isomorphic to the categorical quotient $U(\Sigma) / G_{\Xi}$, where $U(\Sigma)$ is the complement in $\mathbb{C}^{\Xi}$ of the 'bad locus'

$$
Z_{\Sigma}=\bigcup_{\pi \text { primitive family }} \mathbb{A}(\pi), \quad \text { with } \mathbb{A}(\pi):=\left\{z_{\rho}=0, \forall \rho \in \pi\right\} .
$$

We have the following informations concerning $\operatorname{Aut}_{g}(R)$ :

$\underline{\text { Result }}\left[1\right.$, prop. 4.3, 4.6] (i) $\operatorname{Aut}_{g}(R)$ is a connected algebraic group;

(ii) Each $\phi \in \operatorname{Aut}_{g}(R)$ defines an automorphism $\tilde{\phi}$ of $\mathbb{C}^{\Xi}$ having the property that $\tilde{\phi}\left(Z_{\Sigma}\right)=Z_{\Sigma}$.

Actually, the second statement is proved in [1] only for complete and simplicial fans. However, in the appendix A we show that the proof given in loc. cit. extends to complete fans too.

Let us notice that since $Z_{\Sigma}=\bigcup_{\pi \text { primitive }} \mathbb{A}(\pi)$ is the decomposition of $Z_{\Sigma}$ into its irreducible components and since the group $\operatorname{Aut}_{g}(R)$ is connected and preserves $Z_{\Sigma}$, each component $\mathbb{A}(\pi)$ must be preserved under the $\operatorname{Aut}_{g}(R)$-action.

Let us consider now $\xi^{\prime}, \xi^{\prime \prime} \in \Xi$ with $\xi^{\prime} \sim \xi^{\prime \prime}$ and assume that $\pi_{0}$ is a primitive family with $\xi^{\prime} \in \pi_{0}$; we want to prove that $\xi^{\prime \prime}$ still belongs to $\pi_{0}$. Since $c\left(f_{\xi^{\prime}}\right)=$ 
$c\left(f_{\xi^{\prime \prime}}\right)$, the assignment

$$
\begin{aligned}
& \phi\left(Z_{\xi}\right):=Z_{\xi}, \quad \forall \xi \in \Xi \backslash\left\{\xi^{\prime}, \xi^{\prime \prime}\right\}, \\
& \phi\left(Z_{\xi^{\prime}}\right):=Z_{\xi^{\prime \prime}} \quad \text { and } \quad \phi\left(Z_{\xi^{\prime \prime}}\right):=Z_{\xi^{\prime}}
\end{aligned}
$$

extends to a graded automorphism of $R$, that is it defines an element of $\operatorname{Aut}_{g}(R)$. The only effect of the corresponding automorphism $\tilde{\phi}$ of $\mathbb{C}^{\Xi}$ is to exchange the coordinates $z_{\xi^{\prime}}$ and $z_{\xi^{\prime \prime}}$. Let us assume now that $\xi^{\prime \prime} \notin \pi_{0}$. Then, on one hand,

$$
\tilde{\phi}\left(\mathbb{A}\left(\pi_{0}\right)\right)=\tilde{\phi}\left(\left\{z_{\xi}=0 \mid \xi \in \pi_{0}\right\}\right)=\left\{\begin{array}{c}
z_{\xi}=0 \\
z_{\xi^{\prime \prime}}=0
\end{array} \mid \xi \in \pi_{0} \backslash\left\{\xi^{\prime}\right\}\right\},
$$

and, on the other hand, $\mathbb{A}\left(\pi_{0}\right)$ is invariant under the group action

$$
\tilde{\phi}\left(\mathbb{A}\left(\pi_{0}\right)\right)=\mathbb{A}\left(\pi_{0}\right)=\left\{\begin{array}{c}
z_{\xi}=0 \\
z_{\xi^{\prime}}=0
\end{array} \mid \xi \in \pi_{0} \backslash\left\{\xi^{\prime}\right\}\right\} .
$$

Clearly, the two equalities are contradictory, so it must be that $\xi^{\prime \prime} \in \pi_{0}$.

Definition 1.3. For a primitive family $\pi$, we say that an element $\beta \in A_{\Xi}$ is included in $\pi$, and we write $\beta \subset \pi$, if there is a $\xi^{\prime} \in \Xi$ such that $\xi^{\prime} \in \pi$ and $c\left(f_{\xi^{\prime}}\right)=\beta$.

The previous proposition implies that $\beta \subset \pi$ if and only if $\left\{\xi \mid f_{\xi} \in c^{-1}(\beta)\right\} \subset \pi$. In other words, the definition above does make sense.

We pass now to the second part of this section, where we recall the construction of toric varieties as quotients of open subsets in affine spaces. Here we will work in a more general setting than before: $N \cong \mathbb{Z}^{n}$ is going to be a lattice again, but this time $\Xi \subset N$ is a set which satisfies the following properties: (i) $0 \notin \Xi$, (ii) $\sum_{\xi \in \Xi} \mathbb{R}_{\geq 0} \xi=N_{\mathbb{R}}$, (iii) every $\xi \in \Xi$ is the generator over $\mathbb{Z}_{\geq 0}$ of the semi-group $\mathbb{R}_{\geq 0} \xi \cap N$.

We are interested in complete fans $\Sigma \subset N_{\mathbb{R}}$ having the property that the generators of $\Sigma(1)$ form a subset of $\Xi$. Let us fix such a fan $\Sigma$, and denote by $J:=\Xi \backslash \Sigma(1)$ the complement of $\Sigma(1)$ in $\Xi$. In analogy with (1.1), we have this time the commutative diagram

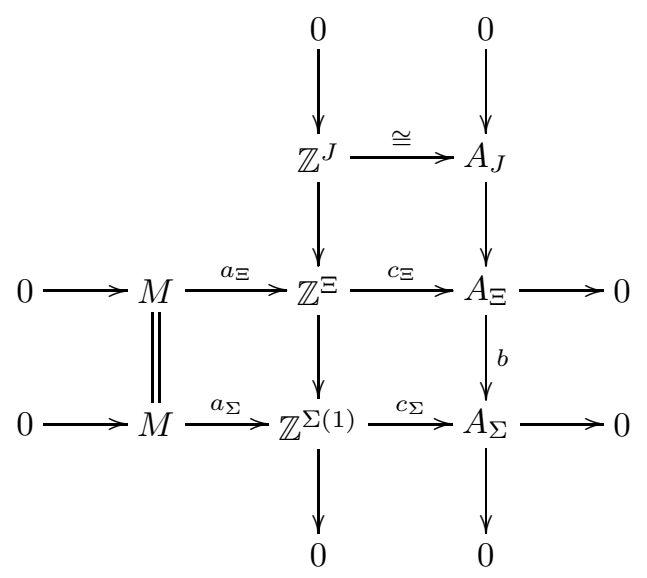


and by dualizing we obtain the commutative diagram of groups

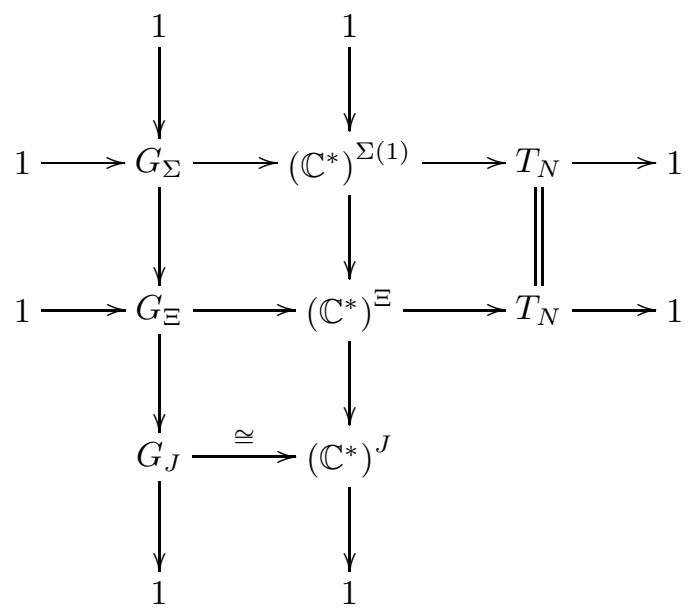

We are interested in describing the decompositions of $\mathbb{C}^{\Xi}$ into the weight spaces corresponding to the $G_{\Xi}$ and $G_{\Sigma}$-actions. The $G_{\Xi \text {-action determines the decompo- }}$ sition

$$
\mathbb{C}^{\Xi}=\bigoplus_{\xi \in \Xi} \mathbb{C} f_{\xi}=\bigoplus_{\alpha \in A_{\Xi}}\left(\bigoplus_{f_{\xi} \in c_{\Xi}^{-1}(\alpha)} \mathbb{C} f_{\xi}\right)=: \bigoplus_{\alpha \in A_{\Xi}} V_{\alpha},
$$

while for the $G_{\Sigma}$-action we have

$$
\mathbb{C}^{\Xi}=\bigoplus_{\beta \in A_{\Sigma}}\left(\bigoplus_{\alpha \in b^{-1}(\beta)} V_{\alpha}\right)=: \bigoplus_{\beta \in A_{\Sigma}} W_{\beta}
$$

We can see from the second diagram that induced $G_{\Sigma}$-action on the $\mathbb{C}^{J}$ summand of $\mathbb{C}^{\Xi}=\mathbb{C}^{\Sigma(1)} \oplus \mathbb{C}^{J}$ is trivial. The following lemma says that $\mathbb{C}^{J}$ can be intrinsically characterized as the trivial isotypic component of $\mathbb{C}^{\Xi}$ corresponding to the $G_{\Sigma^{-}}$ action.

Lemma 1.4. (i) $\mathbb{C}^{\Sigma(1)}=\bigoplus_{\beta \in A_{\Sigma} \backslash\{0\}} W_{\beta}$ and $\mathbb{C}^{J}=W_{0}=\bigoplus_{j \in \operatorname{ker}(b)} V_{j}$;

(ii) Every non-zero weight space $V_{j}$, with $j \in \operatorname{ker}(b)$, has dimension one.

Proof. For the first part it is sufficient to show that there are no linear subspaces of $\mathbb{C}^{\Sigma(1)}$ on which $G_{\Sigma}$ acts trivially. If there was such a linear subspace, acted on trivially by $G_{\Sigma}$, we deduced the existence of a $\xi_{0} \in \Sigma(1)$ with the property that $c_{\Sigma}\left(f_{\xi_{0}}\right)=0$. This would imply the existence of an element $m \in M$ such that $\left\langle m, \xi_{0}\right\rangle=1$ and $\langle m, \xi\rangle=0$ for all $\xi \in \Sigma(1) \backslash\left\{\xi_{0}\right\}$. But this contradicts the assumption that $\Sigma$ is complete.

For the second claim, let us assume the contrary, that there exists an $j \in \operatorname{ker}(b)$ such that $\operatorname{dim} V_{j} \geq 2$. Then, from the first part of this lemma, follows that there are $\eta^{\prime}, \eta^{\prime \prime} \in J$ such that $c_{\Xi}\left(f_{\eta^{\prime}}\right)=c_{\Xi}\left(f_{\eta^{\prime \prime}}\right)=j$. This in turn implies the existence of $m \in M \backslash\{0\}$ such that

$$
\left\langle m, \eta^{\prime}\right\rangle=-\left\langle m, \eta^{\prime \prime}\right\rangle=1 \text { and }\langle m, \xi\rangle=0, \forall \xi \in \Xi \backslash\left\{\eta^{\prime}, \eta^{\prime \prime}\right\} .
$$

In particular, $\langle m, \xi\rangle=0$ for all $\xi \in \Sigma(1)$, and this contradicts that $\Sigma$ is a complete fan. 
Using this language, we can describe more invariantly the 'bad set' in $\mathbb{C}^{\Sigma(1)}$ :

$$
\begin{aligned}
& Z_{\Sigma}=\bigcup_{\pi \text { primitive }} \mathbb{A}(\pi), \quad \text { with } \quad \mathbb{A}(\pi)=\left\{\underline{z}_{\alpha}:=\left(z_{\xi}\right)_{f_{\xi} \in c_{\Sigma}^{-1}(\alpha)}=0, \forall \alpha \subset \pi\right\} \\
& =\operatorname{Ker}\left[\operatorname{pr}: \mathbb{C}^{\Sigma(1)} \rightarrow \oplus_{\beta \subset \pi} W_{\beta}\right]
\end{aligned}
$$

We have already recalled from [1], theorem 2.1, that when $\Xi=\Sigma(1)$, the toric variety $X_{\Sigma}$ can be described as the categorical quotient $U(\Sigma) / G_{\Sigma}$. It is obtained by glueing together the affine pieces $X_{\sigma}=U_{\sigma} / G_{\Sigma}, \sigma \in \Sigma$, where $U_{\sigma}:=\left\{\hat{Z}_{\sigma} \neq 0\right\} \subset$ $\mathbb{C}^{\Sigma(1)}$ and $\hat{Z}_{\sigma}:=\prod_{\xi \in \Sigma(1) \backslash \sigma(1)} Z_{\xi}$.

We want to generalize this situation to our context where the fan $\Sigma$ has the property that $\Sigma(1) \subseteq \Xi$ only. Let us consider the projection $q: \mathbb{C}^{\Xi} \rightarrow \mathbb{C}^{\Sigma(1)}$ corresponding to the decomposition $\mathbb{C}^{\Xi}=\mathbb{C}^{\Sigma(1)} \oplus \mathbb{C}^{J}$, and $W_{0}^{\times}:=\left(\mathbb{C}^{*}\right)^{J} \subset \mathbb{C}^{J}$.

Proposition 1.5. The toric variety $X_{\Sigma}$ is isomorphic to the categorical quotient $\left(U(\Sigma) \times W_{0}^{\times}\right) / G_{\Xi}$. If $\Sigma$ is simplicial, this quotient is geometric.

Proof. What we shall actually prove is that the categorical quotient $(U(\Sigma) \times$ $\left.W_{0}^{\times}\right) / G_{\Xi}$ is isomorphic to $U(\Sigma) / G_{\Sigma}$, and the conclusion will follow then from the result of D. Cox.

The open subset $U(\Sigma) \times W_{0}^{\times} \subset \mathbb{C}^{\Xi}$ is the union of the affine pieces $U_{\sigma} \times W_{0}^{\times}$, and it suffices to check that the quotients $\left(U_{\sigma} \times W_{0}^{\times}\right) / G_{\Xi}$ and $U_{\sigma} / G_{\Sigma}$ are isomorphic, and that these isomorphisms can be glued together on the overlaps. For $\sigma \in \Sigma$ a cone, the ring of functions on $U_{\sigma} \times W_{0}^{\times}$is

$$
\mathbb{C}\left[U_{\sigma} \times W_{0}^{\times}\right]=\mathbb{C}\left[U_{\sigma}\right] \otimes_{\mathbb{C}} \mathbb{C}\left[W_{0}^{\times}\right]=R_{\hat{Z}_{\sigma}} \otimes_{\mathbb{C}} \mathbb{C}\left[Z_{j}, Z_{j}^{-1} \mid j \in J\right]_{Z_{J}},
$$

where the lower indices denote the localizations at $\hat{Z}_{\sigma}=\prod_{\xi \in \Sigma(1) \backslash \sigma(1)} Z_{\xi}$ and $Z_{J}:=$ $\prod_{j \in J} Z_{j}$ respectively. Using the diagram (1.2), we deduce that

$$
\mathbb{C}\left[U_{\sigma} \times W_{0}^{\times}\right]^{G_{\Xi}}=\bigoplus_{m \in \sigma^{\vee} \cap M} \mathbb{C} \cdot \prod_{\rho \in \Xi} Z_{\rho}^{\langle m, \rho\rangle} .
$$

and that

$$
\mathbb{C}\left[U_{\sigma}\right]^{G_{\Sigma}}=\bigoplus_{m \in \sigma^{\vee} \cap M} \mathbb{C} \cdot \prod_{\xi \in \Sigma(1)} Z_{\xi}^{\langle m, \xi\rangle} .
$$

We define the ring homomorphism

$$
\mathbb{C}\left[U_{\sigma} \times W_{0}^{\times}\right]^{G \Xi} \longrightarrow \mathbb{C}\left[U_{\sigma}\right]^{G_{\Sigma}} \text { by } P=P\left(Z_{\xi}, Z_{j}\right) \longmapsto Q_{P}:=P\left(Z_{\xi}, 1\right) .
$$

It is both injective and surjective because $\Sigma$ is complete. The isomorphisms clearly patch over the overlaps, and therefore we deduce the isomorphism of the two categorical quotients.

Let us assume now that $\Sigma$ is simplicial. Then we know that $U(\Sigma) / G_{\Sigma}$ is a geometric quotient. Since for any $\sigma \in \Sigma$,

$$
\mathbb{C}\left[U_{\sigma} \times W_{0}^{\times}\right]^{G_{\Xi}}=\left[\mathbb{C}\left[U_{\sigma} \times W_{0}^{\times}\right]^{G_{\Sigma}}\right]^{G_{\Xi} / G_{\Sigma}=G_{J}}=\left[\mathbb{C}\left[U_{\sigma}\right]^{G_{\Sigma}} \times \mathbb{C} \mathbb{C}\left[W_{0}^{\times}\right]\right]^{G_{J}},
$$

we can decompose the $G_{\Xi-q u o t i e n t}$ into a sequence of two succesive quotients

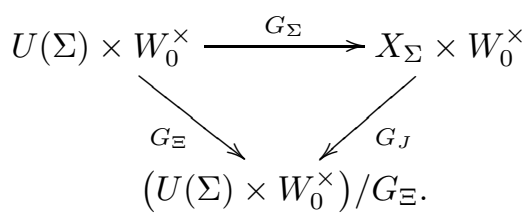


We know that the $G_{\Sigma}$-quotient is geometric, and we notice that $G_{J}$ acts freely and transitively on $W_{0}^{\times} \subset \mathbb{C}^{J} \subset \mathbb{C}^{\Xi}$. Therefore the second quotient is still geometric. Globally, we deduce that the $G_{\Xi}$-quotient is geometric.

We must notice that the isomorphism between the quotients $\left(U(\Sigma) \times W_{0}^{\times}\right) / G_{\Xi}$ and $U(\Sigma) / G_{\Sigma}$ is not natural, and this fact will have as a consequence that the two quotients will give rise, in the relative setting, to distinct families of toric varieties.

Another point that we wish to discuss is related to the sheaves associated to the invariant divisors on a toric variety. It is well-known that given a fan $\Sigma$, to each element $\xi \in \Sigma(1)$ we can associate an irreducible Weil divisor $D_{\xi} \subset X_{\Sigma}$, invariant under the action of the big torus of $X_{\Sigma}$, and any such divisor defines the rank one, coherent sheaf $\mathcal{O}\left(D_{\xi}\right) \rightarrow X_{\Sigma}$. The shortcomming of this construction is that it depends on coordinates, though the sheaf $\mathcal{O}\left(D_{\xi}\right)$ itself does not: for $\xi^{\prime} \sim \xi^{\prime \prime}$, the corresponding sheaves are isomorphic. Our next goal is to describe $\mathcal{O}\left(D_{\xi}\right)$ in a coordinate free fashion, which will eventually allow us to define their analogues in the relative setting.

Proposition 1.6. Let $\Xi \subset N$ be a finite set obeying the conditions (i)-(iii), and let $\Sigma \subset N_{\mathbb{R}}$ be a complete fan with $\Sigma(1) \subseteq \Xi$. Let us denote with $p: U(\Sigma) \rightarrow X_{\Sigma}$ and $r=p q: U(\Sigma) \times W_{0}^{\times} \rightarrow X_{\Sigma}$ the projections and consider $\xi \in \Sigma(1)$. Then:

(i) $\left(p_{*} \mathcal{O}_{U(\Sigma)}\right)^{c_{\Sigma}\left(f_{\xi}\right)}$ and $\left(r_{*} \mathcal{O}_{U(\Sigma) \times W_{0}^{\times}}\right)^{c_{\Xi}\left(f_{\xi}\right)}$ are both isomorphic with $\mathcal{O}\left(D_{\xi}\right)$. If $\Sigma$ is regular, $\mathcal{O}\left(D_{\xi}\right) \cong U(\Sigma) \times_{c_{\Sigma}\left(f_{\xi}\right)} \mathbb{C} \cong\left(U(\Sigma) \times W_{0}^{\times}\right) \times_{c_{\Xi}\left(f_{\xi}\right)} \mathbb{C}$. The sheaves $\left(r_{*} \mathcal{O}_{U(\Sigma) \times W_{0}^{\times}}\right)^{c_{\Xi}\left(f_{j}\right)}, j \in J$, are all isomorphic to $\mathcal{O}_{X_{\Sigma}}$.

(ii) $p^{*}\left(p_{*} \mathcal{O}_{U(\Sigma)}\right) \rightarrow U(\Sigma)$ is isomorphic with $\mathcal{O}_{U(\Sigma)}$, for all $\xi \in \Sigma(1)$. The $G_{\Sigma^{-}}$ action on $U(\Sigma)$ admits a canonical linearization in $p^{*} \mathcal{O}\left(D_{\xi}\right)$, such that for any open subset $O \subset X_{\Sigma}$ the sections of $\mathcal{O}\left(D_{\xi}\right) \rightarrow O$ correspond to the $G_{\Sigma}$-invariant sections in $p^{*} \mathcal{O}\left(D_{\xi}\right) \rightarrow p^{-1} O$. Under the isomorphism above, this linearization correponds to the action with character $c_{\Sigma}\left(f_{\xi}\right)$ of $G_{\Sigma}$ on $\mathcal{O}_{U(\Sigma)}$.

Similarly, $\left.r^{*}\left(r_{*} \mathcal{O}_{U(\Sigma) \times W_{0}^{\times}}\right)^{c_{\Xi}\left(f_{\xi}\right)}\right)$ is isomorphic with $\mathcal{O}_{U(\Sigma) \times W_{0}^{\times}}$, for all $\xi \in \Xi$. The linearization of the $G_{\Xi}$-action corresponds, via this isomorphism to the action with weight $c_{\Xi}\left(f_{\xi}\right)$.

Proof. (i) We determine the sections of $\left(p_{*} \mathcal{O}_{U(\Sigma)}\right)^{c_{\Sigma}\left(f_{\xi}\right)}$ and $\left(r_{*} \mathcal{O}_{U(\Sigma) \times W_{0}^{\times}}\right)^{c_{\Xi}\left(f_{\xi}\right)}$ over the affine pieces $X_{\sigma} \subset X_{\Sigma}$. For $\sigma \in \Sigma$ a cone, we define the sets

$$
M_{\xi}(\sigma)=\left\{\begin{array}{l}
\sigma^{\vee} \cap M=\left\{m \in M \mid\left\langle m, \xi^{\prime}\right\rangle \geq 0, \forall \xi^{\prime} \in \sigma(1)\right\} \quad \text { if } \xi \notin \sigma(1) ; \\
\left\{\begin{array}{ll}
m \in M & \begin{array}{l}
\left\langle m, \xi^{\prime}\right\rangle \geq 0, \forall \xi^{\prime} \in \sigma(1) \backslash\{\xi\} \\
\langle m, \xi\rangle \geq-1
\end{array}
\end{array}\right\} \quad \text { if } \xi \in \sigma(1) .
\end{array}\right.
$$

We can give now explicitely describe the space of sections over $X_{\sigma}$ of the various sheaves which appear in the context:

$$
\begin{aligned}
& \Gamma\left(X_{\sigma},\left(p_{*} \mathcal{O}_{U(\Sigma)}\right)^{c_{\Sigma}\left(f_{\xi}\right)}\right)=\bigoplus_{m \in M_{\xi}(\sigma)} \mathbb{C} \cdot Z_{\xi} \prod_{\xi^{\prime} \in \Sigma(1)} Z_{\xi^{\prime}}^{\left\langle m, \xi^{\prime}\right\rangle} \\
& \Gamma\left(X_{\sigma},\left(r_{*} \mathcal{O}_{U(\Sigma) \times W_{0}^{\times}}\right)^{c_{\Xi}\left(f_{\xi}\right)}\right)=\bigoplus_{m \in M_{\xi}(\sigma)} \mathbb{C} \cdot Z_{\xi} \prod_{\rho \in \Xi} Z_{\rho}^{\langle m, \rho\rangle}, \\
& \Gamma\left(X_{\sigma},\left(r_{*} \mathcal{O}_{U(\Sigma) \times W_{0}^{\times}}\right)^{c_{\Xi}\left(f_{j}\right)}\right)=\bigoplus_{m \in \sigma^{\vee} \cap M} \mathbb{C} \cdot Z_{j} \prod_{\rho \in \Xi} Z_{\rho}^{\langle m, \rho\rangle}, \\
& \Gamma\left(X_{\sigma}, \mathcal{O}_{X_{\Sigma}}\left(D_{\xi}\right)\right)=\bigoplus_{m \in M_{\xi}(\sigma)} \mathbb{C} \cdot \prod_{\xi^{\prime} \in \Sigma(1)} Z_{\xi^{\prime}}^{\left\langle m, \xi^{\prime}\right\rangle}
\end{aligned}
$$


The first three equalities are immediate consequences of the diagram (1.2), while the fourth one can be found in [3, page 66]. We claim that the homomorphism

$$
\left(r_{*} \mathcal{O}_{U(\Sigma)}\right)^{c \Xi\left(f_{\eta}\right)} \longrightarrow \mathcal{O}_{X_{\Sigma}}\left(D_{\xi}\right), \quad Z_{\eta} \prod_{\rho \in \Xi} Z_{\rho}^{\langle m, \rho\rangle} \longmapsto \prod_{\xi^{\prime} \in \Sigma(1)} Z_{\xi^{\prime}}^{\left\langle m, \xi^{\prime}\right\rangle}
$$

defines a sheaf isomorphism for every $\eta \in \Xi=\Sigma(1) \cup J$, since every $m \in M$ is uniquely defined by its evaluation on the elements in $\Sigma(1)$.

For $j \in J$, the function

$$
U(\Sigma) \times W_{0}^{\times} \longrightarrow \mathbb{C}, \quad(u, w) \longmapsto w_{j}
$$

is $G_{\Xi}$-equivariant with respect to the $c_{\Xi}\left(f_{j}\right)$-action on $\mathbb{C}$, and therefore defines a global section $X_{\Sigma} \rightarrow\left(p_{*} \mathcal{O}_{U(\Sigma) \times W_{0}^{\times}}\right)^{c \Xi\left(f_{j}\right)}$ which vanishes nowhere. Moreover, the description of the section spaces given above above implies that $\left(p_{*} \mathcal{O}_{U(\Sigma) \times W_{0}^{\times}}\right)^{c_{\Xi}\left(f_{j}\right)}$ is locally free of rank one. It is therefore isomorphic to $\mathcal{O}_{X_{\Sigma}}$.

(ii) We prove our statements for $p^{*}\left(p_{*} \mathcal{O}_{U(\Sigma)}\right) \rightarrow U(\Sigma)$, since the other case is completely analogous. We distinguish again between two possibilities, according to whether $\xi$ belongs to $\sigma(1)$ or not. For $\xi \notin \sigma(1)$,

$$
\begin{aligned}
\mathbb{C}\left[U_{\sigma}\right] \otimes_{\mathbb{C}\left[U_{\sigma}\right]^{G_{\Sigma}}} \Gamma\left(X_{\sigma},\left(p_{*} \mathcal{O}_{U(\Sigma)}\right)^{c_{\Sigma}\left(f_{\xi}\right)}\right) & =\mathbb{C}\left[U_{\sigma}\right] \otimes_{\mathbb{C}\left[U_{\sigma}\right]^{G_{\Sigma}}} \mathbb{C}\left[U_{\sigma}\right]^{G_{\Sigma}} \cdot Z_{\xi} \\
& =\mathbb{C}\left[U_{\sigma}\right] \otimes_{\mathbb{C}} \mathbb{C} Z_{\xi},
\end{aligned}
$$

and we notice that in this case $Z_{\xi}$ is invertible in $\mathbb{C}\left[U_{\sigma}\right]$.

On the other hand, the smallest invariant open set $U_{\sigma} \subset U(\Sigma)$ with the property that $\xi \in \sigma(1)$ is $U_{\xi}:=U_{\mathbb{R}_{\geq 0} \xi}$ (let us recall that $\mathbb{R}_{\geq 0} \xi$ is itself a cone of $\Sigma)$. Then, if we fix some $m_{0} \in M_{\xi}\left(\mathbb{R}_{\geq 0} \xi\right)$ such that $\left\langle m_{0}, \xi\right\rangle=-1$, and write $Z^{m_{0}}:=\prod_{\xi^{\prime} \in \Sigma(1)} Z_{\xi^{\prime}}^{\left\langle m_{0} \cdot \xi^{\prime}\right\rangle}$, we find that

$$
\begin{aligned}
\mathbb{C}\left[U_{\xi}\right] \otimes_{\mathbb{C}\left[U_{\xi}\right]^{G_{\Sigma}}} \Gamma\left(X_{\xi},\left(p_{*} \mathcal{O}_{U(\Sigma)}\right)^{c_{\Sigma}\left(f_{\xi}\right)}\right) & =\mathbb{C}\left[U_{\xi}\right] \otimes_{\mathbb{C}\left[U_{\xi}\right]^{G_{\Sigma}}} \mathbb{C}\left[U_{\xi}\right]^{G_{\Sigma}} \cdot Z_{\xi} Z^{m_{0}} \\
& =\mathbb{C}\left[U_{\xi}\right] \otimes_{\mathbb{C}} \mathbb{C} Z_{\xi} Z^{m_{0}} .
\end{aligned}
$$

Again, since $\left\langle m_{0}, \xi\right\rangle=-1, Z_{\xi} Z^{m_{0}}$ is invertible in $\mathbb{C}\left[U_{\xi}\right]$. Consequently,

$$
p^{*}\left[\left(p_{*} \mathcal{O}_{U(\Sigma)}\right)^{c_{\Sigma}\left(f_{\xi}\right)}\right] \stackrel{\text { product }}{\longrightarrow} \mathcal{O}_{U(\Sigma)}
$$

is an isomorphism of modules. Since $G_{\Sigma}$ acts on both $Z_{\xi}$ and $Z_{\xi} Z^{m_{0}}$ with character $c_{\Sigma}\left(f_{\xi}\right)$, we deduce that the induced linearization is given by the weight $c_{\Sigma}\left(f_{\xi}\right)$.

Let us remark that for any $\beta \in A_{\Sigma}$ and $\xi \in \Sigma(1)$ such that $c_{\Sigma}\left(f_{\xi}\right)=\beta$,

$$
\Gamma\left(X_{\Sigma},\left(p_{*} \mathcal{O}_{U(\Sigma)}\right)^{c_{\Sigma}\left(f_{\xi}\right)}\right)=R_{\beta}
$$

the summand of degree $\beta$ of the homogeneous ring $R$. Moreover, $R_{\beta}$ decomposes into the direct sum $R_{\beta}^{\prime} \oplus R_{\beta}^{\prime \prime}$, where $R_{\beta}^{\prime}=\oplus_{\left\{\xi \mid f_{\xi} \in c_{\Sigma}^{-1}(\beta)\right\}} \mathbb{C} Z_{\xi}$ and $R_{\beta}^{\prime \prime}$ consists of sums of monomials of degree $\beta$ which are products of two or more variables. In the terminology of [1, section 4], $R_{\beta}^{\prime}$ corresponds to the semi-simple roots of $X_{\Sigma}$, while $R_{\beta}^{\prime \prime}$ corresponds to the unipotent roots.

The point of this discussion, which we are going to use in the next section, is that $W_{\beta}^{\vee}$ is canonically isomorphic to $R_{\beta}^{\prime}$, and therefore is a direct summand in $\Gamma\left(X_{\Sigma},\left(p_{*} \mathcal{O}_{U(\Sigma)}\right)^{c_{\Sigma}\left(f_{\xi}\right)}\right)$. 
Let us consider now the ring $\tilde{R}:=\mathbb{C}\left[Z_{\rho} \mid \rho \in \Xi\right]$. The group $G_{\Xi}$ acts on it, and the corresponding weights define a graduation

$$
\tilde{R}=\bigoplus_{\alpha \in A_{\Xi}} \tilde{R}_{\alpha}
$$

Similarly, for any $\alpha \in A_{\Xi}$ and $\xi \in \Xi$ such that $c_{\Xi}\left(f_{\xi}\right)=\alpha$,

$$
\Gamma\left(X_{\Sigma},\left(r_{*} \mathcal{O}_{U(\Sigma) \times W_{0}^{\times}}\right)^{c_{\Xi}\left(f_{\xi}\right)}\right)=\tilde{R}_{\alpha},
$$

and $\tilde{R}_{\alpha}=\tilde{R}_{\alpha}^{\prime} \oplus \tilde{R}_{\alpha}^{\prime \prime}$, with $\tilde{R}_{\alpha}^{\prime}$ canonically isomorphic to $V_{\alpha}^{\vee}$.

Now we fix $\xi \in \Sigma(1)$ and we wish to understand the relationship between $\tilde{R}_{c \Xi\left(f_{\xi}\right)}$ and $R_{c_{\Sigma}\left(f_{\xi}\right)}$. Let us define

$$
M_{\xi}:=\left\{m \in M \mid \begin{array}{l}
\langle m, \xi\rangle \geq-1 \\
\left\langle m, \xi^{\prime}\right\rangle \geq 0, \forall \xi^{\prime} \in \Sigma(1) \backslash\{\xi\}
\end{array}\right\} .
$$

Then

$$
\begin{aligned}
& \Gamma\left(X_{\Sigma}, \tilde{\mathscr{L}}_{\xi}\right)=\oplus_{m \in M_{\xi}} \mathbb{C} Z_{\xi} \cdot \prod_{\rho \in \Xi} Z_{\rho}^{\langle m, \rho\rangle} \\
& \Gamma\left(X_{\Sigma}, \mathscr{L}_{\xi}\right)=\oplus_{m \in M_{\xi}} \mathbb{C} Z_{\xi} \cdot \prod_{\xi^{\prime} \in \Sigma(1)}^{\left\langle m, \xi^{\prime}\right.} Z_{\xi^{\prime}}
\end{aligned}
$$

and we notice that

$$
\begin{gathered}
\tilde{R}_{c_{\Xi}\left(f_{\xi}\right)}=\Gamma\left(X_{\Sigma}, \tilde{\mathscr{L}}_{\xi}\right) \stackrel{f}{\longrightarrow} \Gamma\left(X_{\Sigma}, \mathscr{L}_{\xi}\right)=R_{c_{\Sigma}\left(f_{\xi}\right)} \\
Z_{\xi} \cdot \prod_{\rho \in \Xi} Z_{\rho}^{\langle m, \rho\rangle} \longmapsto Z_{\xi} \cdot \prod_{\xi^{\prime} \in \Sigma} Z_{\xi^{\prime}}^{\left\langle m, \xi^{\prime}\right\rangle}
\end{gathered}
$$

is an isomorphism of modules, as it should be, since $\tilde{\mathscr{L}}_{\xi} \cong \mathcal{O}_{X_{\Sigma}}\left(D_{\xi}\right) \cong \mathscr{L}_{\xi}$.

We want to determine $f^{-1} R_{c_{\Sigma}\left(f_{\xi}\right)}^{\prime}$. For shorthand, we write $\beta=c_{\Sigma}\left(f_{\xi}\right)$. First of all, $R_{\beta}^{\prime}=\oplus_{\left\{\xi^{\prime} \mid f_{\xi^{\prime}} \in c_{\Sigma}^{-1}(\beta)\right\}} \mathbb{C} Z_{\xi^{\prime}}$, by definition. We partition the indices as follows

$$
\left\{\xi^{\prime} \mid f_{\xi^{\prime}} \in c_{\Sigma}^{-1}(\beta)\right\}=\bigsqcup_{\alpha \in b^{-1}(\beta)}\left\{\xi^{\prime} \mid c_{\Xi}\left(f_{\xi^{\prime}}\right)=\alpha\right\} .
$$

Lemma 1.7. For every $\alpha \in b^{-1}(\beta)$, there are integers $\left(k_{j}(\alpha)\right)_{j \in J}$ depending on $\alpha$, such that for all $f_{\xi^{\prime}} \in c_{\Xi}^{-1}(\alpha)$ holds: $f^{-1} Z_{\xi^{\prime}}=Z_{\xi^{\prime}} \prod_{j \in J} Z_{j}^{k_{j}(\alpha)}$.

Proof. Let us fix such $\alpha$ and $\xi_{0}^{\prime}$ : then $c_{\Sigma}\left(f_{\xi_{0}^{\prime}}\right)=\beta=c_{\Sigma}\left(f_{\xi}\right)$, and therefore we find a unique $m_{\xi_{0}^{\prime}} \in M$ such that $\left\langle m_{\xi_{0}^{\prime}}, \xi\right\rangle=-1,\left\langle m_{\xi_{0}^{\prime}}, \xi_{0}^{\prime}\right\rangle=+1$, and all the other evaluations on vectors in $\Sigma(1) \backslash\left\{\xi, \xi_{0}^{\prime}\right\}$ vanish. Then $Z_{\xi_{0}^{\prime}}=Z_{\xi} \cdot \prod_{\xi^{\prime} \in \Sigma(1)} Z_{\xi^{\prime}}^{\left\langle m, \xi^{\prime}\right\rangle}$ and $f^{-1} Z_{\xi_{0}^{\prime}}=Z_{\xi} \cdot \prod_{\rho \in \Xi} Z_{\rho}^{\left\langle m_{\xi_{0}^{\prime}}, \rho\right\rangle}=Z_{\xi_{0}^{\prime}} \prod_{j \in J} Z_{j}^{\left\langle m_{\xi_{0}^{\prime}}, j\right\rangle}$. We define $k_{j}(\alpha):=\left\langle m_{\xi_{0}^{\prime}}, j\right\rangle$, and we must check that these numbers do not depend on the choice of $\xi_{0}^{\prime}$ satisfying $c_{\Xi}\left(f_{\xi_{0}^{\prime}}\right)=\alpha$. If $\xi_{0}^{\prime \prime}$ is another vector with the same property, we find a unique $m_{\xi_{0}^{\prime} \xi_{0}^{\prime \prime}} \in M$ which evaluates -1 on $\xi_{0}^{\prime}$, +1 on $\xi_{0}^{\prime \prime}$, and zero on the other vectors in $\Xi \backslash\left\{\xi_{0}^{\prime}, \xi_{0}^{\prime \prime}\right\}$. From the unicity of the elements $\left\{m_{\xi^{\prime}}\right\}_{\xi^{\prime}}$, we deduce that $m_{\xi_{0}^{\prime \prime}}=$ $m_{\xi_{0}^{\prime}}+m_{\xi_{0}^{\prime} \xi_{0}^{\prime \prime}}$, and the evaluations of it on $j \in J$ coincide with those of $m_{\xi_{0}^{\prime}}$.

The lemma can now be reformulated as

$$
f^{-1} R_{\beta}^{\prime}=\bigoplus_{\alpha \in b^{-1}(\beta)} \tilde{R}_{\alpha} \otimes_{\mathbb{C}} \mathbb{C} \prod_{j \in J} Z_{j}^{k_{j}(\alpha)}=\bigoplus_{\alpha \in b^{-1}(\beta)} V_{\alpha}^{\vee} \otimes_{\mathbb{C}} \mathbb{C} \prod_{j \in J}\left(V_{j}^{\vee}\right)^{\otimes k_{j}(\alpha)},
$$

and this is the form that we will need subsequently. 


\section{The CONSTRUCtion}

This section is devoted presenting a method for constructing families of complete toric varieties, parameterized by some base space. The idea behind the construction is to replace $\mathbb{C}^{\Xi}$, which should be seen as the as the total space of a vector bundle over a point, with an arbitrary vector bundle $\mathcal{V} \rightarrow S$ on which a torus acts. The result of the construction should be a locally trivial family of toric varieties, parameterized by the base $S$.

Let us make things more precise: the main ingredients are again the lattice $N$ and the collection of vectors $\Xi \subset N$ having the properties (i)-(iii) enumerated in the previous section. Associated to this data we have the exact sequence

$$
1 \longrightarrow G_{\Xi} \longrightarrow\left(\mathbb{C}^{*}\right)^{\Xi} \longrightarrow T_{N} \longrightarrow 1 \text {. }
$$

Corresponding to the induced $G_{\Xi}$-action, we obtain the decomposition of $\mathbb{C}^{\Xi}$ into the $G_{\Xi}$-isotypic components

$$
\mathbb{C}^{\Xi}=\bigoplus_{\alpha \in A \Xi} V_{\alpha}, \quad \operatorname{dim} V_{\alpha}=r_{\alpha}
$$

The second ingredient for the construction is a separated, noetherian scheme $S$ and a collection of arbitrary vector bundles (locally free sheaves) $\mathcal{V}_{\alpha} \rightarrow S$ of rank $r_{\alpha}$ respectively. We define the vector bundle

$$
\mathcal{V}:=\bigoplus_{\alpha \in A_{\Xi}} \underline{\mathbb{C}}_{\alpha} \otimes \mathcal{V}_{\alpha}
$$

where $\mathbb{C}_{\alpha}$ stands for the trivial vector bundle over $S$ on which $G_{\Xi}$ acts by the character $\alpha$. For this choice of the vector bundle $\mathcal{V} \rightarrow S$, we obtain fibrewise the same $G_{\Xi}$-action as the one described in the preceeding section.

At this point we recall a result concerning the structure of vector bundles with a linear group action, which will be needed later on.

$\underline{\text { Result }}[7$, prop. 1] Let $G$ be a reductive group and $\mathcal{V} \rightarrow S$ a $G$-vector bundle, where $G$ acts trivially on $S$.

(i) $\mathcal{V} \rightarrow S$ is locally trivial, as a G-vector bundle, in the Zariski topology. In particular, if $S$ is connected, all the representations $G \rightarrow G l\left(\mathcal{V}_{s}\right), s \in S$, are equivalent.

(ii) There is an isomorphism of G-vector bundles

$$
\nu \stackrel{\cong}{\longrightarrow} \bigoplus_{\omega} M_{\omega} \otimes \mathcal{V}_{\omega}
$$

where the $M_{\omega}$ 's are simple $G$-modules and the $\mathcal{V}_{\omega}$ 's are vector bundles with trivial $G$-action. The direct summands $M_{\omega} \otimes \mathcal{V}_{\omega}$ are called the $\omega$-isotypic components of $\mathcal{V}$, and they are uniquely defined.

We choose now a complete fan $\Sigma \subset N_{\mathbb{R}}$ with $\Sigma(1) \subseteq \Xi$ and we want to construct a locally trivial fibration over $S$ with all the fibres isomorphic to the toric variety $X_{\Sigma}$. Since $G_{\Sigma}$ is a subgroup of $G_{\Xi}$, the vector bundle $\mathcal{V}$ gets an induced $G_{\Sigma^{-}}$ action. For every $\alpha \in A_{\Xi}, G_{\Sigma}$ acts on $\underline{\mathbb{C}}_{\alpha} \otimes \mathcal{V}_{\alpha}$ by the character $b(\alpha) \in A_{\Sigma}$, where the homomorphism $b: A_{\Xi} \rightarrow A_{\Sigma}$ was defined in (1.2). We deduce that the $G_{\Sigma}$-isotypical decomposition of $\mathcal{V}$ is

$$
\mathcal{V}=\bigoplus_{\beta \in A_{\Sigma}} \mathcal{W}_{\beta}=\left(\bigoplus_{\beta \in A_{\Sigma} \backslash\{0\}} \mathcal{W}_{\beta}\right) \oplus \mathcal{W}_{0}=: \mathcal{W}_{\Sigma} \oplus \mathcal{W}_{0}
$$


In this formula, for each $\beta \in A_{\Sigma}$, we have denoted $\mathcal{W}_{\beta}:=\bigoplus_{\alpha \in b^{-1}(\beta)} \underline{\mathbb{C}}_{\alpha} \otimes \mathcal{V}_{\alpha}$.

Lemma 2.1. $\mathcal{W}_{0}=\bigoplus_{\alpha \in \operatorname{ker}(b)} \underline{\mathbb{C}}_{\alpha} \otimes \mathcal{V}_{\alpha} \rightarrow S$ is a vector bundle whose rank equals $\#(\Xi \backslash \Sigma(1))$. Moreover, each of the $\underline{\mathbb{C}}_{\alpha} \otimes \mathcal{V}_{\alpha}$ 's has rank equal one.

Proof. By the first part of the result above, $\mathcal{V}$ is locally trivial, as a $G_{\Sigma}$-bundle, in the Zariski topology. Therefore, locally over $S$, we are in the situation of the lemma 1.4 and the conclusion follows.

Definition 2.2. We shall write $\mathcal{W}_{0}^{\times}:=\bigoplus_{j \in \operatorname{Ker}(b)}\left[\left(\mathbb{C}_{j} \otimes \mathcal{V}_{j}\right) \backslash\{0\}\right]$. For a $\Sigma$-primitive family $\pi \subseteq \Sigma(1)$, we define the subvector bundle

$$
\mathcal{W}(\pi):=\operatorname{Ker}\left[\operatorname{pr}_{\pi}: \mathcal{W}_{\Sigma} \longrightarrow \bigoplus_{\beta \subset \pi} \mathcal{W}_{\beta}\right] \subset \mathcal{W}_{\Sigma} .
$$

The meaning of the notation $\beta \subset \pi$ was defined in 1.3.

We denote by $z_{\Sigma}$ the union of these bundles as $\pi$ varies among the $\Sigma$-primitive families

$$
z_{\Sigma}:=\bigcup_{\pi \text { primitive }} \mathcal{W}(\pi) \subset \mathcal{W}_{\Sigma}
$$

Theorem 2.3. Let us consider $\Sigma \subset N_{\mathbb{R}}$ a complete fan with $\Sigma(1) \subseteq \Xi$, and define the open subsets $\mathscr{U}(\Sigma):=\mathcal{W}_{\Sigma} \backslash \mathcal{Z}_{\Sigma} \subset \mathcal{W}_{\Sigma}$ and $\tilde{\mathscr{U}}(\Sigma):=\mathscr{U}(\Sigma) \times_{S} \mathcal{W}_{0}^{\times} \subset \mathcal{V}$. Then the categorical quotients $\tilde{\mathscr{U}}_{\Sigma} / G_{\Xi}$ and $\mathscr{U}(\Sigma) / G_{\Sigma}$ does exist. They are locally trivial fibrations over $S$, with the fibres isomorphic to the toric variety $X_{\Sigma}$ defined by the fan $\Sigma$. When $\Sigma$ is moreover simplicial, the above quotients are geometric.

Proof. We shall prove the statement concerning $\tilde{\mathscr{U}}(\Sigma)$, the proof for $\mathscr{U}(\Sigma)$ being completely identical.

The first thing to notice is that $\tilde{\mathscr{U}}(\Sigma)$ is $G_{\Xi}$-invariant, since $\mathscr{U}(\Sigma)$ is $G_{\Sigma^{-}}$ invariant. We know that the $G_{\Xi}$-bundle $\mathcal{V}$ is locally trivial in the Zariski topology. In order to construct the desired quotient we can restrict ourselves to open subsets of $S$ : let us choose some open subset $O \subset S$ over which $\mathcal{V}$ is trivializable. It follows from (1.4) that $\left.\tilde{\mathscr{U}}(\Sigma)\right|_{O} \cong O \times\left(U(\Sigma) \times W_{0}^{\times}\right)$, where $U(\Sigma)=\mathbb{C}^{\Sigma(1)} \backslash Z_{\Sigma}$ (see section 1 ), the isomorphism being $G_{\Xi}$-equivariant. We use now proposition 1.5 and deduce the existence of the categorical quotient $\left.\tilde{\mathscr{U}}(\Sigma)\right|_{O} / G_{\Xi}$, which is isomorphic with $O \times X_{\Sigma}$. From the universality property of the categorical quotient, it follows that, as $O \subset S$ varies, we can paste these quotients together. Therefore the categorical quotient $\tilde{\mathscr{U}}(\Sigma) / G_{\Sigma}$ exists, and it is a $X_{\Sigma}$-fibration over the base $S$.

When $\Sigma$ is simpicial, we know that $\left(U(\Sigma) \times W_{0}^{\times}\right) / G_{\Xi}$ is a geometric quotient, and consequently, $\tilde{\mathscr{U}}(\Sigma) / G_{\Xi}$ is still geometric.

Remark 2.4. The most classical example of such a family is the projectivized bundle of an arbitrary vector bundle over some base variety.

We must clearly point out that the two quotients $\tilde{\mathscr{U}}(\Sigma) / G_{\Xi}$ and $\mathscr{U}(\Sigma) / G_{\Sigma}$ are not isomorphic in general. Of course, the case $\Sigma(1)=\Xi$ is an exception, since then the two families coincide. The reason for obtaining in general different fibrations is due to the fact that the isomorphism between $\left(U(\Sigma) \times W_{0}^{\times}\right) / G_{\Xi}$ and $U(\Sigma) / G_{\Sigma}$ described in the proposition 1.5 was not natural. When $\Sigma$ is simplicial, we will see in the next section that we can distinguish them even at the cohomological level. 
The families $\tilde{\mathscr{X}}_{\Sigma}$ and $\mathscr{X}_{\Sigma}$ are related in the following fashion: let us take the quotient $\tilde{\mathscr{U}}(\Sigma) / G_{\Xi}$ in two steps, first for the $G_{\Sigma}$-action and then for the remaining $G_{J}=G_{\Xi} / G_{\Sigma}$-action. We deduce that

$$
\tilde{\mathscr{X}}_{\Sigma}=\left(\mathscr{X}_{\Sigma} \times_{S} \mathcal{W}_{0}^{\times}\right) / G_{J}
$$

and this quotient is always geometric since $\mathcal{W}_{0}^{\times} \rightarrow S$ is a principal $G_{J}$-bundle.

We shall denote with $\tilde{\mathscr{X}}_{\Sigma} \rightarrow S$ and $\mathscr{X}_{\Sigma} \rightarrow S$ respectively the two families constructed above. For a given pair $(N, \Xi)$ and a given collection of vector bundles $\left\{\mathcal{V}_{\alpha} \rightarrow S\right\}_{\alpha}$, we remark that both $\tilde{\mathscr{X}}_{\Sigma}$ and $\mathscr{X}_{\Sigma}$ depend on the choice of the complete fan $\Sigma \subset N_{\mathbb{R}}$ with $\Sigma(1) \subseteq \Xi$. There are several ways of choosing complete fans satisfying this property, and the detailed account about this issue can be found in [8]. The moral of the discussion is that one may construct several different fibrations out of a given vector bundle $\mathcal{V} \rightarrow S$ with a given $G_{\Xi}$-action.

We are going now to take advantage of the invariant, coordinate-free description of the sheaves $\mathcal{O}_{X_{\Sigma}} \rightarrow X_{\Sigma}$, and define their analogues for families of toric varieties. Before doing this, we recall from lemma 1.4 that for $j \in J, V_{j} \rightarrow S$ is a line bundle.

Proposition 2.5. Let $\Sigma$ be a complete fan with $\Sigma(1) \subseteq \Xi$. For each $\xi \in \Sigma(1)$ we define

$$
\tilde{\mathscr{L}}_{\xi}:=\left(r_{*} \mathcal{O}_{\tilde{\mathscr{U}}(\Sigma)}\right)^{c_{\Xi}\left(f_{\xi}\right)} \longrightarrow \tilde{\mathscr{X}}_{\Sigma} \text { and } \mathscr{L}_{\xi}:=\left(p_{*} \mathcal{O}_{\mathscr{U}(\Sigma)}\right)^{c_{\Sigma}\left(f_{\xi}\right)} \longrightarrow \mathscr{X}_{\Sigma},
$$

where $r: \tilde{\mathscr{U}}(\Sigma) \rightarrow \tilde{\mathscr{X}}_{\Sigma}$ and $p: \mathscr{U}(\Sigma) \rightarrow \mathscr{X}_{\Sigma}$ are the projections.

(i) $\tilde{\mathscr{L}}_{\xi}$ and $\mathscr{L}_{\xi}$ are coherent, torsion free sheaves of rank one. Their restiction to the fibres of the corresponding families are isomorphic with $\mathcal{O}_{X_{\Sigma}}\left(D_{\xi}\right)$. Moreover, $\tilde{\mathscr{L}}_{\xi}$ and $\mathscr{L}_{\xi}$ are locally free outside a singular set of codimension at least two.

When $\Sigma$ is regular, both sheaves are locally free:

$$
\tilde{\mathscr{L}}_{\xi}=\tilde{\mathscr{U}}(\Sigma) \times_{c_{\Xi}\left(f_{\xi}\right)} \mathbb{C} \text { and } \mathscr{L}_{\xi}=\mathscr{U}(\Sigma) \times_{c_{\Sigma}\left(f_{\xi}\right)} \mathbb{C} .
$$

(ii) $r^{*} \tilde{\mathscr{L}}_{\xi} \rightarrow \tilde{\mathscr{U}}(\Sigma)$ and $p^{*} \mathscr{L}_{\xi} \rightarrow \mathscr{U}(\Sigma)$ are respectively isomorphic to $\mathcal{O}_{\tilde{\mathscr{U}}(\Sigma)}$ and $\mathcal{O}_{\mathscr{U}(\Sigma)}$, and they carry natural $G_{\Xi}$ and $G_{\Sigma}$-linearizations. These linearizations correpond respectively, under the isomorphisms, to the actions with weights $c_{\Xi}\left(f_{\xi}\right)$ and $c_{\Sigma}\left(f_{\xi}\right)$.

(iii) For $j \in J, \tilde{\mathscr{L}}_{j}:=\left(r_{*} \mathcal{O}_{\tilde{\mathscr{U}}(\Sigma)}\right)^{c \Xi\left(f_{j}\right)} \longrightarrow \tilde{\mathscr{X}}_{\Sigma}$ is isomorphic to $\tilde{\phi}^{*} V_{j}^{\vee}$, where $\tilde{\phi}$ : $\tilde{\mathscr{X}}_{\Sigma} \rightarrow S$ is the projection. Moreover, $r^{*} \tilde{\mathscr{L}}_{j} \cong \mathcal{O}_{\tilde{\mathscr{X}}_{\Sigma}}$, and the induced linearization of $G_{\Xi}$ correponds to the action with weight $c_{\Xi}\left(f_{j}\right)$.

Proof. (i) Again, we shall treat only the case of $\tilde{\mathscr{L}}_{\xi}$. Let us consider an open subset $O \subset S$ having the property that $\left.\mathcal{V}\right|_{O} \rightarrow O$ is trivializable. The proposition 1.6 implies that over such trivializing open subsets

$$
\left.\tilde{\mathscr{L}}_{\xi}\right|_{O} \cong O \times \mathcal{O}_{X_{\Sigma}}\left(D_{\xi}\right) \longrightarrow O \times X_{\Sigma}
$$

Is is clearly a torsion-free sheaf, and its rank equals one. Since $\mathcal{O}_{X_{\Sigma}}\left(D_{\xi}\right) \rightarrow X_{\Sigma}$ is coherent, and the coherence property is local in nature, we deduce the coherence of $\tilde{\mathscr{L}}_{\xi} \rightarrow \tilde{\mathscr{X}}_{\Sigma}$. Moreover, since $X_{\Sigma}$ is a normal variety (according to [3, page 29]), we deduce that $\mathcal{O}_{X_{\Sigma}}\left(D_{\xi}\right) \rightarrow X_{\Sigma}$ is locally free outside a closed subset of codimension at least two in $X_{\Sigma}$. The isomorphism above implies that the same holds for $\tilde{\mathscr{L}}_{\xi} \rightarrow \tilde{\mathscr{X}}_{\Sigma}$. 
(ii) The germ of $r^{*} \tilde{\mathscr{L}}_{\xi}$ at a point $\tilde{u} \in \tilde{\mathscr{U}}(\Sigma)$ is

$$
\left(\tilde{\mathscr{L}}_{\xi}\right)_{\tilde{u}}=\lim _{r(\tilde{u}) \in O}\left(\mathcal{O}_{\tilde{\mathscr{U}}(\Sigma)}\left(r^{-1} O\right) \otimes_{\mathcal{O}_{\tilde{\mathscr{X}}_{\Sigma}}(O)}\left(\mathcal{O}_{\tilde{\mathscr{U}}(\Sigma)}\left(r^{-1} O\right)\right)^{c_{\Xi}\left(f_{\xi}\right)}\right),
$$

and we find the natural homomorphism

$$
\left(\tilde{\mathscr{L}}_{\xi}\right)_{\tilde{u}} \stackrel{\text { product }}{\longrightarrow} \lim _{r(\tilde{u}) \in O} \mathcal{O}_{\tilde{\mathscr{U}}(\Sigma)}\left(r^{-1} O\right) \longrightarrow \mathcal{O}_{\tilde{\mathscr{U}}(\Sigma), \tilde{u}}
$$

In other words, there is a well-defined a homomorphism $r^{*} \tilde{\mathscr{L}}_{\xi} \rightarrow \mathcal{O}_{\tilde{\mathscr{U}}(\Sigma)}$, and we have checked in proposition 1.6 that it is actually an isomorphism. The statement about the linearizations is proved in the same place.

(iii) For the last part, let us choose a trivializing covering $\left\{O_{\nu}\right\}_{\nu}$ of $S$, for $\mathcal{V}_{j}$, having the property that there are non-vanishing sections $s_{\nu}:\left.\mathcal{O}_{S}\left(O_{\nu}\right) \rightarrow \mathcal{V}_{j}\right|_{O_{\nu}}$. Then $\mathcal{V}_{j}$ is associated to the co-cycle $\left\{s_{\mu}^{-1} s_{\nu}\right\}_{\nu, \mu}$. Let $\operatorname{pr}_{s}: \tilde{\mathscr{U}}(\Sigma) \rightarrow S$ be the projection. Then we have a nowhere vanishing section

$$
s_{j}: \tilde{\mathscr{U}}(\Sigma) \longrightarrow \operatorname{pr}_{S}^{*} \mathcal{V}_{j}, \quad(u, w) \longmapsto\left((u, w), w_{j}\right) .
$$

For each $\nu$, the composition

$$
s_{j, \nu}:\left.\left.\tilde{\mathscr{U}}(\Sigma)\right|_{O_{\nu}} \stackrel{s_{j}}{\longrightarrow} \operatorname{pr}_{S}^{*} \mathcal{V}_{j}\right|_{O_{\nu}} \stackrel{\operatorname{pr}_{S}^{*} s_{\nu}^{-1}}{\longrightarrow} \operatorname{pr}_{S}^{*} \mathcal{O}_{S}\left(O_{\nu}\right)=\left.\mathcal{O}_{\tilde{\mathscr{U}}(\Sigma)}\right|_{O_{\nu}}
$$

is $G_{\Xi}$-equivariant and non-vanishing, and therefore defines a trivializing section $\left.\left.\tilde{\mathscr{X}}_{\Sigma}\right|_{O_{\nu}} \rightarrow \tilde{\mathscr{L}}_{j}\right|_{O_{\nu}}$. We deduce that the co-cycle defining $\tilde{\mathscr{L}}_{j}$ is $\left\{s_{j, \mu}^{-1} s_{j, \nu}\right\}_{\nu, \mu}$, with

$$
s_{j, \mu}^{-1} s_{j, \nu}=\tilde{\phi}^{*}\left(s_{\mu}^{-1} s_{\nu}\right)=\tilde{\phi}^{*}\left(\left(s_{\mu}^{-1} s_{\nu}\right)^{-1}\right),
$$

so that $\tilde{\mathscr{L}}_{j} \cong \tilde{\phi}^{*} \mathcal{V}_{j}^{-1}$. The statement about the linearizations follows from the same proposition 1.6 .

It is a well known fact that for a projective bundle $\mathbb{P}(\mathcal{W}) \rightarrow S$, the direct image on $S$ of $\mathcal{O}_{\mathbb{P}(\mathcal{W})}(1)$ is isomorphic to $\mathcal{W}^{\vee}$. We wish to investigate now what happens in our more general setting, that is we wish to compute the direct images on $S$ of the sheaves $\tilde{\mathscr{L}}_{\xi}$ and $\mathscr{L}_{\xi}$.

Let us denote by $\mathcal{P}_{\Xi}$ and $\mathcal{P}_{\Sigma}$ respectively the principal bundles of frames in $\mathcal{V} \rightarrow S$ and $\mathcal{W}_{\Sigma} \rightarrow S$ : the structure group of $\mathcal{P}_{\Xi}$ is $\mathcal{G}\left(\mathcal{P}_{\Xi}\right):=\prod_{\alpha \in A \Xi} G l\left(V_{\alpha}\right)$, while the structure group of $\mathcal{P}_{\Sigma}$ is $\mathcal{G}\left(\mathcal{P}_{\Sigma}\right):=\prod_{\beta \in A_{\Sigma}} G l\left(W_{\beta}\right)$.

Proposition 2.6. (i) Consider $\xi \in \Sigma(1)$ and denote $c_{\Xi}\left(f_{\xi}\right)=\alpha$ and $c_{\Sigma}\left(f_{\xi}\right)=\beta$. Then

and contains

$$
\tilde{\phi}_{*} \tilde{\mathscr{L}}_{\xi} \cong \mathcal{P} \times_{\mathcal{G}(\mathcal{P} \equiv)} \tilde{R}_{\alpha}, \quad \text { for } \tilde{\phi}: \tilde{\mathscr{X}}_{\Sigma} \rightarrow S,
$$

$$
\bigoplus_{\alpha^{\prime} \in b^{-1}(\beta)}\left[\mathcal{V}_{\alpha^{\prime}}^{\vee} \otimes\left(\otimes_{j \in J} \mathcal{V}_{j}^{-k_{j}\left(\alpha^{\prime}\right)}\right)\right]
$$

as a direct summand. For $j \in J, \tilde{\phi}_{*} \tilde{\mathscr{L}}_{j} \cong \mathcal{V}_{j}^{\vee}$.

(ii) For every $\beta \in A_{\Sigma}$ and $\xi \in \Sigma(1)$ with $c_{\Sigma}\left(f_{\xi}\right)=\beta$,

$$
\phi_{*} \mathscr{L}_{\xi} \cong \mathcal{P}_{\Sigma} \times_{\mathcal{G}\left(\mathcal{P}_{\Sigma}\right)} \tilde{R}_{\alpha}, \quad \text { for } \phi: \mathscr{X}_{\Sigma} \rightarrow S,
$$

and contains $\mathcal{W}_{\beta}^{\vee}$ as a direct summand.

In particular, if $X_{\Sigma}$ has no unipotent roots, $\tilde{\phi}_{*} \tilde{\mathscr{L}}_{\xi}$ and $\phi_{*} \mathscr{L}_{\xi}$ are isomorphic respectively to $\bigoplus_{\alpha^{\prime} \in b^{-1}(\beta)}\left[\mathcal{V}_{\alpha^{\prime}}^{\vee} \otimes\left(\otimes_{j \in J} \mathcal{V}_{j}^{-k_{j}\left(\alpha^{\prime}\right)}\right)\right]$ and $W_{\beta}^{\vee}$. 
Proof. Since for all $j \in J, \tilde{\mathscr{L}}_{j} \cong \tilde{\phi}^{*} \mathcal{V}_{j}^{\vee}$ and $X_{\Sigma}$ is connected, $\phi_{*} \tilde{\mathscr{L}}_{j} \cong \mathcal{V}_{j}^{\vee}$.

Let us choose a covering $\left\{O_{\nu}\right\}_{\nu}$ of $S$ which is simultaneously trivializing for the bundles $\left\{\mathcal{V}_{\alpha}\right\}_{\alpha \in A_{\Xi}}$. It is also a trivializing covering for the bundles $\mathcal{P}_{\Xi}$ and $\mathcal{P}_{\Sigma}$, and the corresponding transition maps $\left\{\tilde{g}_{\mu \nu}\right\}_{\mu, \nu}$ and $\left\{g_{\mu \nu}\right\}_{\mu, \nu}$ are elements in $\mathcal{G}\left(\mathcal{P}_{\Xi}\right)$ and $\mathcal{G}\left(\mathcal{P}_{\Sigma}\right)$ respectively. We observe that these transition functions define automorphisms of $\tilde{U}(\Sigma):=U(\Sigma) \times W_{0}^{\times}$and $U(\Sigma)$ respectively, which in turn induce automorphisms of $X_{\Sigma}$ (cf. [1, section 4]). We notice further that since $\left\{\tilde{g}_{\mu \nu}\right\}$ and $\left\{g_{\mu \nu}\right\}$ respect the graduation of the rings $\tilde{R}$ and $R$ respectively, they induce automorphisms of $\left(r_{*} \mathcal{O}_{\tilde{U}(\Sigma)}\right)^{c_{\Xi}\left(f_{\xi}\right)}$ and $\left(p_{*} \mathcal{O}_{U(\Sigma)}\right)^{c_{\Sigma}\left(f_{\xi}\right)}$ such that the diagrams

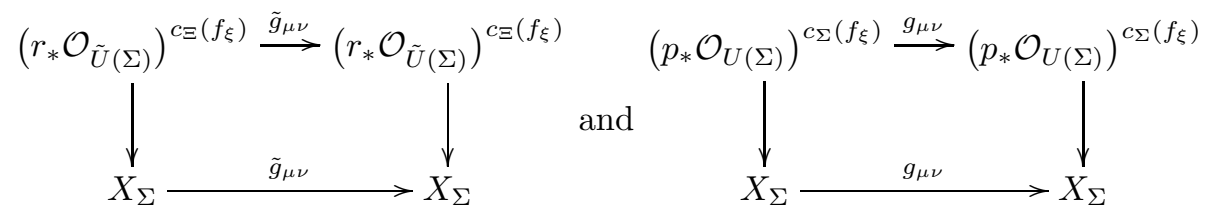

commute. It follows then that $\tilde{\mathscr{X}}_{\Sigma}=\mathcal{P}_{\Xi} \times_{\mathcal{G}\left(\mathcal{P}_{\Xi}\right)} X_{\Sigma}$ and $\mathscr{X}_{\Sigma}=\mathcal{P}_{\Sigma} \times_{\mathcal{G}\left(\mathcal{P}_{\Sigma}\right)} X_{\Sigma}$. Moreover, above the trivializing open sets we have the isomorphisms $\left.\tilde{\phi}_{*} \tilde{\mathscr{L}}_{\xi}\right|_{O_{\nu}} \cong$ $O_{\nu} \times \tilde{R}_{\alpha}$ and $\left.\phi_{*} \mathscr{L}_{\xi}\right|_{O_{\nu}} \cong O_{\nu} \times R_{\beta}$, and from what we said we deduce that $\tilde{\phi}_{*} \tilde{\mathscr{L}}_{\xi}$ and $\phi_{*} \mathscr{L}_{\xi}$ are the the vector bundles associated to $\mathcal{P}_{\Xi}$ and $\mathcal{P}_{\Sigma}$ for the representations $\mathcal{G}\left(\mathcal{P}_{\Xi}\right) \rightarrow G l\left(\tilde{R}_{\alpha}\right)$ and $\mathcal{G}\left(\mathcal{P}_{\Sigma}\right) \rightarrow G l\left(R_{\beta}\right)$ respectively. This proves the first half of the statements (i) and (ii).

For the second half, we have proved in the end of section 1 that $W_{\beta}^{\vee}=R_{\beta}^{\prime}$ is direct summand in $R_{\beta}$, and the equation (1.5) shows that $f^{-1} R_{\beta}^{\prime}=\bigoplus_{\alpha \in b^{-1}(\beta)} V_{\alpha}^{\vee} \otimes_{\mathbb{C}}$ $\mathbb{C} \prod_{j \in J}\left(V_{j}^{\vee}\right)^{\otimes k_{j}(\alpha)}$ is direct summand in $\tilde{R}_{\alpha}$. Moreover, these sub-vector spaces are stable under the actions of the structure groups $\mathcal{G}\left(\mathcal{P}_{\Sigma}\right)$ and $\mathcal{G}(\mathcal{P})$ respectively. This proves the rest of the proposition.

Let us assume now that $S$ is projective. We want to prove that in this case the sheaves $\tilde{\mathscr{L}}_{\xi} \rightarrow \tilde{\mathscr{X}}_{\Sigma}$ and $\mathscr{L}_{\xi} \rightarrow \mathscr{X}_{\Sigma}$ define elements $\left[\tilde{\mathscr{L}}_{\xi}\right] \in A^{1}\left(\tilde{\mathscr{X}}_{\Sigma}\right)$ and $\left[\mathscr{L}_{\xi}\right] \in A^{1}\left(\mathscr{X}_{\Sigma}\right)$. We have proved in proposition 2.5 that both $\tilde{\mathscr{L}}_{\xi}$ and $\mathscr{L}_{\xi}$ are locally free outside $\tilde{\mathscr{X}}_{\Sigma \text {,bad }} \hookrightarrow \tilde{\mathscr{X}}_{\Sigma}$ and $\mathscr{X}_{\Sigma \text {,bad }} \hookrightarrow \mathscr{X}_{\Sigma}$ respectively, whose codimension is at least two. Since $S$ is projective we can choose a sufficiently ample line bundle $A \rightarrow S$ having the property that $\tilde{\phi}_{*} \tilde{\mathscr{L}}_{\xi} \otimes A, \phi_{*} \mathscr{L}_{\xi} \otimes A$ and $A$ are all globally generated over $S$. Let us choose regular sections

$$
\begin{aligned}
& \tilde{s} \in \Gamma\left(S, \tilde{\phi}_{*} \tilde{\mathscr{L}}_{\xi} \otimes A\right)=\Gamma\left(\tilde{\mathscr{X}}_{\Sigma}, \tilde{\mathscr{L}}_{\xi} \otimes \tilde{\phi}^{*} A\right), \\
& s \in \Gamma\left(S, \phi_{*} \mathscr{L}_{\xi} \otimes A\right)=\Gamma\left(\mathscr{X}_{\Sigma}, \mathscr{L}_{\xi} \otimes \tilde{\phi}^{*} A\right) \text { and } s_{A} \in \Gamma(S, A),
\end{aligned}
$$

which do not identically vanish on any irreducible component of $S$.

Using them we can associate to the restrictions $\left.\tilde{\mathscr{L}}_{\xi}\right|_{\tilde{X}_{\Sigma} \backslash \tilde{\mathscr{X}}_{\Sigma, \text { bad }}}$ and $\left.\mathscr{L}_{\xi}\right|_{\mathscr{X}_{\Sigma} \backslash \mathscr{X}_{\Sigma, \text { bad }}}$ the classes $\left[\left.\tilde{\mathscr{L}}_{\xi}\right|_{\left.\tilde{\mathscr{X}}_{\Sigma} \backslash \tilde{\mathscr{X}}_{\Sigma, \text { bad }}\right]}\right]:=[\operatorname{Zero}(\tilde{s})]-\left[\operatorname{Zero}\left(\tilde{\phi}^{*} s_{A}\right)\right] \in A^{1}\left(\tilde{\mathscr{X}}_{\Sigma} \backslash \tilde{\mathscr{X}}_{\Sigma, \text { bad }}\right)$ and $\left[\left.\mathscr{L}_{\xi}\right|_{\mathscr{X}_{\Sigma} \backslash \mathscr{X}_{\Sigma, \mathrm{bad}}}\right]:=[\operatorname{Zero}(s)]-\left[\operatorname{Zero}\left(\phi^{*} s_{A}\right)\right] \in A^{1}\left(\mathscr{X}_{\Sigma} \backslash \mathscr{X}_{\Sigma, \mathrm{bad}}\right)$. These classes do not depend on the choice of the sections $\tilde{s}, s$ and $s_{A}$. We recall now that $\tilde{\mathscr{X}}_{\Sigma \text {,bad }} \hookrightarrow \tilde{\mathscr{X}}_{\Sigma}$ and $\mathscr{X}_{\Sigma \text {,bad }} \hookrightarrow \mathscr{X}_{\Sigma}$ both have codimension at least two, and therefore

$$
A^{1}\left(\tilde{\mathscr{X}}_{\Sigma}\right) \longrightarrow A^{1}\left(\tilde{\mathscr{X}}_{\Sigma} \backslash \tilde{\mathscr{X}}_{\Sigma, \mathrm{bad}}\right) \text { and } A^{1}\left(\mathscr{X}_{\Sigma}\right) \longrightarrow A^{1}\left(\mathscr{X}_{\Sigma} \backslash \mathscr{X}_{\Sigma, \mathrm{bad}}\right)
$$

are isomorphisms (cf. [4, proposition 1.8]). We should further notice that these classes depend only on $c_{\Xi}\left(f_{\xi}\right) \in A_{\Xi}$ and $c_{\Sigma}\left(f_{\xi}\right) \in A_{\Sigma}$, and not of $\xi$ itself. 


\section{Cohomology}

In this section we determine the cohomology rings of the families of toric varieties constructed in the previous section, in the case when the fibre is simplicial and complete. This means that our context is as follows: $N$ is a lattice, $\Xi \subset N$ is a finite set satisfying the properties (i)-(iii), and $\Sigma \subset N_{\mathbb{R}}$ is a complete and simplicial fan with $\Sigma(1) \subseteq \Xi$. Associated to this data we have the commutative diagrams (1.2) and (1.3).

The goal of this section is to determine the cohomology rings of the families $\tilde{\mathscr{X}}_{\Sigma}$ and $\mathscr{X}_{\Sigma}$ constructed in the previous section. In particular, we shall see that they are usually not isomorphic, and can be distinguished by cohomological means.

We start with some well-known facts concerning classifying spaces for groups.

Definition 3.1. We denote by $E$ a contractible space on which the group $\left(\mathbb{C}^{*}\right)^{\Xi}$ acts freely. It is uniquely defined up to homotopy equivalence. The quotients

$$
\mathrm{BG}_{\Xi}:=E / G_{\Xi} \text { and } \mathrm{BG}_{\Sigma}:=E / G_{\Sigma}
$$

are called classifying spaces for $G_{\Xi}$ and $G_{\Sigma}$ respectively.

Lemma 3.2.

$$
\begin{aligned}
H^{*}\left(\mathrm{BG}_{\Xi} ; \mathbb{Q}\right) & =\operatorname{Sym}^{\bullet}\left(A_{\Xi}\right)_{\mathbb{Q}} \cong \mathbb{Q}\left[f_{\xi} \mid \xi \in \Xi\right] /\langle\mathrm{LR}\rangle_{\Xi} ; \\
H^{*}\left(\mathrm{BG}_{\Sigma} ; \mathbb{Q}\right) & =\operatorname{Sym}^{\bullet}\left(A_{\Sigma}\right)_{\mathbb{Q}} \cong \mathbb{Q}\left[f_{\xi} \mid \xi \in \Sigma(1)\right] /\langle\mathrm{LR}\rangle_{\Sigma} \\
& \cong H^{*}\left(\mathrm{BG}_{\Xi} ; \mathbb{Q}\right) /\left(\left\langle f_{j} \mid j \in J\right\rangle+\langle\mathrm{LR}\rangle_{\Xi}\right),
\end{aligned}
$$

where $\langle\mathrm{LR}\rangle_{\Xi}:=\left\langle a_{\Xi}(m) \mid m \in M\right\rangle \subset \mathbb{Q}\left[f_{\xi} \mid \xi \in \Xi\right]$, and similarly for $\langle\mathrm{LR}\rangle_{\Sigma}$. We call them the ideals of linear relations. Moreover, if $A_{\Xi}$ has no torsion, both isomorphisms hold with integral coefficients.

For shorthand, we write $H_{G_{\Xi}}^{*}$ and $H_{G_{\Sigma}}^{*}$ respectively for the rings $H^{*}\left(\mathrm{BG}_{\Xi} ;-\right)$ and $H^{*}\left(\mathrm{BG}_{\Sigma} ;-\right)$, where the coefficient ring is $\mathbb{Z}$ or $\mathbb{Q}$, depending on whether $A_{\Xi}$ has torsion or has not.

Let us remark at this point that if there exists a regular fan $\Sigma$ with $\Sigma(1) \subset \Xi$, then $A_{\Xi}$ has no torsion automatically. Indeed, $b:\left(A_{\Xi}\right)_{\text {tor }} \rightarrow\left(A_{\Sigma}\right)_{\text {tor }}$ is always an isomorphism, and for regular fans $\left(A_{\Sigma}\right)_{\text {tor }}=0$.

Proof. The torsions $\left(A_{\Xi}\right)_{\text {tor }} \subset A_{\Xi}$ and $\left(A_{\Sigma}\right)_{\text {tor }} \subset A_{\Sigma}$ are finite abelian groups and $b:\left(A_{\Xi}\right)_{\text {tor }} \rightarrow\left(A_{\Sigma}\right)_{\text {tor }}$ is an isomorphism. The quotients $A_{\Xi}^{o}:=A_{\Xi} /\left(A_{\Xi}\right)_{\text {tor }}$ and $A_{\Sigma}^{o}:=A_{\Sigma} /\left(A_{\Sigma}\right)_{\text {tor }}$ are free $\mathbb{Z}$-module and their duals $G_{\Xi}^{o}$ and $G_{\Sigma}^{o}$ are respectively the connected components of the identity in $G_{\Xi}$ and $G_{\Sigma}$. These latter groups are respectively isomorphic to the direct products $G_{\Xi}^{o} \times F$ and $G_{\Sigma}^{o} \times F$, with $F:=$ $\operatorname{Hom}_{\mathbb{Z}}\left(\left(A_{\Sigma}\right)_{\text {tor }}, \mathbb{C}^{*}\right)$. The classifying spaces for $G_{\Xi}^{o}$ and $G_{\Xi}^{o}$ are obtained as $\mathrm{BG}_{\Xi}^{o}:=$ $E / G_{\Xi}^{o}$ and $\mathrm{BG}_{\Sigma}^{o}:=E / G_{\Sigma}^{o}$ respectively, and they fit in the commutative diagram

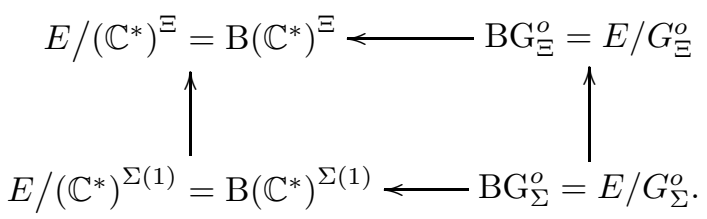

It is known that for a complex torus, the cohomology of its classifying space is naturally isomorphic to the symmetric algebra of its character group. In our case, 
the previous diagram induces

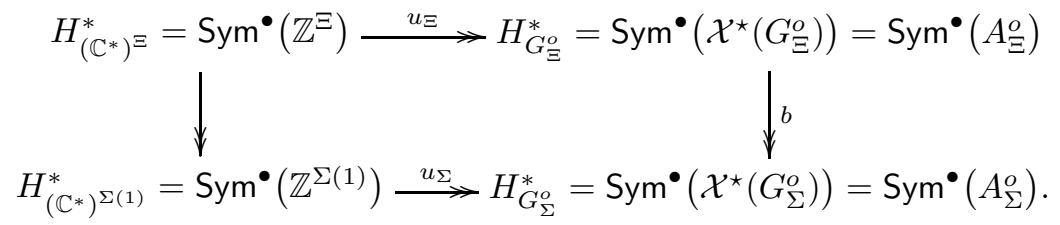

The homomorphisms $u_{\Xi}$ and $u_{\Sigma}$ are surjective and

$$
\operatorname{Ker}\left(u_{\Xi}\right)=\left\langle\operatorname{Ker}\left(c_{\Xi}^{o}\right)\right\rangle, \quad \operatorname{Ker}\left(u_{\Sigma}\right)=\left\langle\operatorname{Ker}\left(c_{\Sigma}^{o}\right)\right\rangle,
$$

with $c_{\Xi}^{o}: \mathbb{Z}^{\Xi} \longrightarrow A_{\Xi}^{o}$ and similarly $c_{\Sigma}^{o}$. If $\left(A_{\Xi}\right)_{\text {tor }}=0$, we are done because in this case $G_{\Xi}$ and $G_{\Sigma}$ coincide with $G_{\Xi}^{o}$ and $G_{\Sigma}^{o}$ respectively, and $\left\langle\operatorname{Ker}\left(c_{\Xi}^{o}\right)\right\rangle=\langle\mathrm{LR}\rangle_{\Xi}$, $\left\langle\operatorname{Ker}\left(c_{\Sigma}^{o}\right)\right\rangle=\langle\mathrm{LR}\rangle_{\Sigma}$.

In general, we notice that

$$
E / G_{\Xi}^{o}=\mathrm{BG}_{\Xi}^{o} \stackrel{\gamma_{\Xi}}{\longrightarrow} E / G_{\Xi}=\mathrm{BG}_{\Xi} \text { and } E / G_{\Sigma}^{o}=\mathrm{BG}_{\Sigma}^{o} \stackrel{\gamma_{\Sigma}}{\longrightarrow} E / G_{\Sigma}=\mathrm{BG}_{\Sigma}
$$

are principal $F$-bundles, and they induce isomorphism in the cohomology with rational coefficients. Then

$$
H^{*}\left(\mathrm{BG}_{\Xi} ; \mathbb{Q}\right) \stackrel{\gamma^{*}}{\cong} H^{*}\left(\mathrm{BG}_{\Xi}^{o} ; \mathbb{Q}\right) \cong \operatorname{Sym}^{\bullet} \mathbb{Q}^{\Xi} /\left\langle\operatorname{Ker}\left(u_{\Xi, \mathbb{Q}}\right)\right\rangle
$$

and we are done again.

Our main tool for computing the cohomology of $\mathscr{X}_{\Sigma}$ is the Leray-Hirsch theorem. For the confort of the reader, we recall this classical result:

Leray-Hirsch theorem $[10$, theorem 5.7.9] Let $p:(\mathcal{F}, \dot{\mathcal{F}}) \rightarrow S$ be the total pair of a fibre-bundle pair with base $S$ and fibre pair $(F, \dot{F})$, and let $R$ be a ring. Assume that $H^{*}(F, \dot{F} ; R)$ is free over $R$ and that $\theta: H^{*}(F, \dot{F} ; R) \rightarrow H^{*}(\mathcal{F}, \dot{\mathcal{F}} ; R)$ is a cohomology extension of the fibre. Then the homomorphism

$$
\begin{gathered}
\Theta^{*}: H^{*}(S ; R) \otimes H^{*}(F, \dot{F} ; R) \longrightarrow H^{*}(\mathcal{F}, \dot{\mathcal{F}} ; R), \\
u \otimes v \longmapsto p^{*} u \cup \theta(v)
\end{gathered}
$$

is an isomorphism of graded modules over $R$.

We should notice that in loc. cit. there is the additional assumption that $H^{*}(F, \dot{F} ; R)$ is finitely generated over $R$. However, according to [6, page 232], this hypothesis can be omitted.

As an immediate consequence, we can determine the ring structure of such a fibre-bundle pair

$$
H^{*}(\mathcal{F}, \dot{\mathcal{F}} ; R) \cong H^{*}(S ; R)\left[\theta(v) \mid v \in H^{*}(F, \dot{F} ; R)\right],
$$

where in this writing the relations among the $\theta(v)$ 's are understood.

We need also the following fact concerning the cohomology ring of complete and simplicial toric varieties:

$\underline{\text { Result }}\left[2\right.$, theorem 10.8 and remark 10.9] Let $X_{\Sigma}$ be a complete and simplicial toric variety. For each $\xi \in \Sigma(1)$ we denote $D_{\xi}$ the corresponding invariant divisor on $X_{\Sigma}$. Then the ring homomorphism

$$
\mathbb{Q}\left[f_{\xi} \mid \xi \in \Sigma(1)\right] \longrightarrow H^{*}\left(X_{\Sigma} ; \mathbb{Q}\right)
$$

induced by the assignment

$$
f_{\xi} \longmapsto\left[D_{\xi}\right] \in H^{2}\left(X_{\Sigma} ; \mathbb{Q}\right)
$$


is surjective. Its kernel is $\langle\mathrm{LR}\rangle_{\Sigma}+\langle\mathrm{SR}\rangle_{\Sigma}$, where

$\langle\mathrm{LR}\rangle_{\Sigma}$ is the ideal of linear relations defined in lemma 3.2, and

$\langle\mathrm{SR}\rangle_{\Sigma}:=\left\langle\prod_{\xi \in \pi} f_{\xi}\right| \pi \subset \Sigma(1)$ is a primitive family $\rangle$ is the Stanley-Reisner ideal.

If $X_{\Sigma}$ is smooth, that is $\Sigma$ is regular, the result holds with integral coefficients.

We shall use again a shorthand notation: we write $H^{*}\left(X_{\Sigma}\right)$ for the cohomology ring of $X_{\Sigma}$ with coefficients in $\mathbb{Z}$ or $\mathbb{Q}$, depending respectively on whether $\Sigma$ is regular or only simplicial.

In view of the lemma 3.2 , we deduce that

$$
h_{\Sigma}: H_{G_{\Sigma}}^{*} \longrightarrow H^{*}\left(X_{\Sigma}\right), \quad \hat{f}_{\xi} \longmapsto\left[D_{\xi}\right]
$$

is a surjective ring homomorphism and $\operatorname{ker}\left(h_{\Sigma}\right)=\langle\mathrm{SR}\rangle_{\Sigma}$. Therefore the composition

$$
h: H_{G_{\Xi}}^{*} \stackrel{b}{\longrightarrow} H_{G_{\Sigma}}^{*} \stackrel{h_{\Sigma}}{\longrightarrow} H^{*}\left(X_{\Sigma}\right)
$$

is still surjective with kernel

$$
\operatorname{Ker}(h)=\left\langle f_{j} \mid j \in J\right\rangle+\langle\mathrm{SR}\rangle_{\Sigma} \subset H_{G_{\Xi}}^{*} .
$$

We can further say that the cohomology of $X_{\Sigma}$ is generated by the first Chern classes of the rank one sheaves $\mathcal{O}\left(D_{\xi}\right) \rightarrow X_{\Sigma}, \xi \in \Sigma(1)$. Proposition 1.6 gives a coordinate-free description for these sheaves as

$$
\mathcal{O}\left(D_{\xi}\right)=\left(r_{*} \mathcal{O}_{U(\Sigma) \times W_{0}^{\times}}\right)^{c_{\Xi}\left(f_{\xi}\right)},
$$

for $r: U(\Sigma) \times W_{0}^{\times} \rightarrow X_{\Sigma}$ the projection. Since $\Sigma$ is simplicial, $r$ is a geometric quotient, and we have a natural ring isomorphism $H^{*}\left(X_{\Sigma}\right) \stackrel{\cong}{\longrightarrow} H_{G_{\Xi}}^{*}(U(\Sigma))$. It follows now from the second half of proposition 1.6 that the diagram

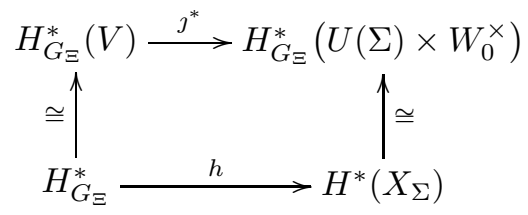

commutes, so that $\jmath^{*}$ is surjective as $h$ is surjective. This fact, together with the long exact sequence in cohomology for the pair $(V, U(\Sigma))$ imply that we have a short exact sequence

$$
0 \longrightarrow H_{G_{\Xi}}^{*}\left(V, U(\Sigma) \times W_{0}^{\times}\right) \stackrel{\imath^{*}}{\longrightarrow} H_{G_{\Xi}}^{*}(V) \stackrel{\jmath^{*}}{\longrightarrow} H_{G_{\Xi}}^{*}\left(U(\Sigma) \times W_{0}^{\times}\right) \longrightarrow 0,
$$

and $\imath^{*}$ and $\jmath^{*}$ are ring homomorphisms. We deduce now that

$$
\imath^{*} H_{G \Xi}^{*}\left(V, U(\Sigma) \times W_{0}^{\times}\right) \subset H_{G_{\Xi}}^{*}(V)
$$

is an ideal. In view of the previous discission, this ideal is precisely

$$
\begin{aligned}
\imath^{*} H_{G_{\Xi}}^{*}\left(V, U(\Sigma) \times W_{0}^{\times}\right)= & \left.\left\langle\prod_{\xi \in \pi} \hat{f}_{\xi}\right| \pi \text { primitive family }\right\rangle+\left\langle\hat{f}_{j} \mid j \in J\right\rangle \\
= & \left.\left\langle e_{G_{\Xi}}\left(W_{\pi}\right)\right| \pi \text { primitive family and } W_{\pi}:=\bigoplus_{\beta \subset \pi} W_{\beta}\right\rangle \\
& +\left\langle e_{G_{\Xi}}\left(V_{j}\right) \mid j \in J\right\rangle .
\end{aligned}
$$


In this formula, $e_{G_{\Xi}}(\cdot)$ stays for the equivariant Euler class of the correponding vector space, and we have used that

$$
e_{G_{\Xi}}\left(W_{\pi}\right)=\prod_{\beta \subset \pi}\left[\prod_{\alpha \in b^{-1}(\beta)} e_{G_{\Xi}}\left(V_{\alpha}\right)\right]=\prod_{\beta \subset \pi}\left[\prod_{\alpha \in b^{-1}(\beta)}\left(\prod_{c \Xi\left(f_{\xi}\right)=\alpha} \hat{f}_{\xi}\right)\right] \stackrel{1.2}{=} \prod_{\xi \in \pi} \hat{f}_{\xi} .
$$

The conclusion of our discussion can now be formulated as

Corollary 3.3. The classes $\left(\imath^{*}\right)^{-1} e_{G_{\Xi}}\left(W_{\pi}\right)$, where $\pi \subset \Sigma(1)$ ranges over the primitive families, and $\left(\imath^{*}\right)^{-1} e_{G_{\Xi}}\left(V_{j}\right), j \in J$, generate $H_{G_{\Xi}}^{*}(V, U(\Sigma))$ as a $H_{G_{\Xi}}^{*}$-algebra.

We should point out that is not so easy to compute the explicit formulae for these generators.

Proposition 3.4. The Leray-Hirsch theorem does apply to the fibration

$$
\begin{gathered}
X_{\Sigma} \hookrightarrow \tilde{\mathscr{X}}_{\Sigma} \\
\downarrow \\
S .
\end{gathered}
$$

Proof. We must show that there does exist a cohomology extension of the fibre $X_{\Sigma}$. We have just seen that the cohomology of $X_{\Sigma}$ is generated by the first Chern classes of the sheaves $\mathcal{O}\left(D_{\xi}\right), \xi \in \Sigma(1)$, and that they can be described by (3.3). In the relative setting, for each $\xi \in \Sigma(1)$, we have defined in proposition 2.5 the rank one sheaf

$$
\tilde{\mathscr{L}}_{\xi}:=\left(r_{*} \mathcal{O}_{\tilde{\mathscr{U}}(\Sigma)}\right)^{c \Xi\left(f_{\xi}\right)},
$$

where $r: \mathscr{U}(\Sigma) \rightarrow \tilde{\mathscr{X}}_{\Sigma}$ is the projection, whose restriction to the fibres of $\tilde{\mathscr{X}}_{\Sigma} \rightarrow S$ is isomorphic to $\mathcal{O}\left(D_{\xi}\right)$.

We claim that the sheaves $\tilde{\mathscr{L}}_{\xi}$ possess a first Chern class. Indeed, we have proved in proposition 2.5 that $\tilde{\mathscr{L}}_{\xi}$ is locally free outside of a subvariety $\tilde{\mathscr{X}}_{\Sigma \text {,bad }} \hookrightarrow \tilde{\mathscr{X}}_{\Sigma}$

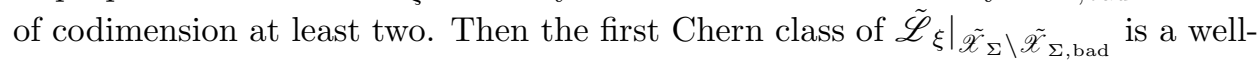
defined element of $H^{2}\left(\tilde{\mathscr{X}}_{\Sigma} \backslash \tilde{\mathscr{X}}_{\Sigma, \text { bad }} ; \mathbb{Z}\right)$, and this group is isomorphic to $H^{2}\left(\tilde{\mathscr{X}}_{\Sigma} ; \mathbb{Z}\right)$ since the bad set has complex codimension at least two in $\tilde{\mathscr{X}}_{\Sigma}$.

Corollary 3.5. The cohomology ring of $\tilde{\mathscr{X}}_{\Sigma}$ is

$$
H^{*}\left(\tilde{\mathscr{X}}_{\Sigma}\right) \cong H^{*}(S)\left[l_{\xi} \mid \xi \in \Sigma(1)\right], \quad \text { with } \quad l_{\xi}:=c_{1}\left(\tilde{\mathscr{L}}_{\xi}\right) \in H^{2}\left(\mathscr{X}_{\Sigma} ; \mathbb{Z}\right) .
$$

In particular, the ring homomorphism

$$
\begin{gathered}
h_{S}: H_{G_{\Xi}}^{*}(S)=H^{*}(S) \otimes H_{G_{\Xi}}^{*} \longrightarrow H^{*}\left(\tilde{\mathscr{X}}_{\Sigma}\right), \\
u \otimes \hat{f}_{\rho} \longmapsto \tilde{\phi}^{*} u \cup l_{\rho}, \forall \rho \in \Xi,
\end{gathered}
$$

is surjective, where $\tilde{\phi}: \tilde{\mathscr{X}}_{\Sigma} \rightarrow S$ is the projection.

Before entering into the proof, we should clarify the notation: for $\rho=j \in J \subset \Xi$, we know from proposition 2.5 that $\tilde{\mathscr{L}}_{j} \cong \tilde{\phi}^{*} \mathcal{V}_{j}^{\vee}$, and therefore $l_{j}=-\tilde{\phi}^{*}\left[\mathcal{V}_{j}\right]$.

Proof. All we need to prove is that the homomorphism $h_{S}$ is well-defined. Since $H_{G_{\Xi}}^{*}$ is the quotient $H_{\left(\mathbb{C}^{*}\right)}^{*} /\langle\mathrm{LR}\rangle_{\Xi}$, we must check that as soon as the classes $\left\{\hat{f}_{\rho}\right\}_{\rho}$ satisfy a linear relation, the classes $\left\{l_{\rho}\right\}_{\rho}$ fulfill the same one.

So, let us consider integers $\left\{a_{\rho}\right\}_{\rho}$ having the property that $\sum_{\rho} a_{\rho} \hat{f}_{\rho}=0$. This is equivalent saying that $\sum_{\rho} a_{\rho} c_{\Xi}\left(f_{\rho}\right)=\sum_{\rho} c_{\Xi}\left(a_{\rho} f_{\rho}\right)=0 \in \mathcal{X}^{\star}\left(G_{\Xi}\right)$. We are going to 
prove that $\bigotimes_{\rho} \tilde{\mathscr{L}}_{\rho}^{\otimes a_{\rho}} \cong \mathcal{O}_{\tilde{\mathscr{X}}_{\Sigma}}$ over $\tilde{\mathscr{X}}_{\Sigma} \backslash \tilde{\mathscr{X}}_{\Sigma \text {,bad }}$, and the conclusion follows then by a codimension two argument.

Let us consider an open subset $O \subset \tilde{\mathscr{X}}_{\Sigma} \backslash \tilde{\mathscr{X}}_{\Sigma \text {,bad }}$, and non-vanishing sections $s_{\rho} \in \Gamma\left(O, \tilde{\mathscr{L}}_{\rho}\right)=\Gamma\left(r^{-1} O, \mathcal{O}_{\tilde{\mathscr{U}}(\Sigma)}\right)^{c \Xi\left(f_{\rho}\right)}$. Then $s:=\bigotimes_{\rho} s_{\rho}^{\otimes a_{\rho}} \in \Gamma\left(r^{-1} O, \mathcal{O}_{\tilde{\mathscr{U}}(\Sigma)}\right)^{\sum_{\rho} c_{\Xi}\left(a_{\rho} f_{\rho}\right)=0}=\Gamma\left(r^{-1} O, \mathcal{O}_{\tilde{\mathscr{U}}(\Sigma)}\right)^{G \Xi}=\Gamma\left(O, \mathcal{O}_{\tilde{\mathscr{X}}_{\Sigma}}\right)$.

The last equality is implied by the fact that $\tilde{\mathscr{X}}_{\Sigma}$ is a geometric quotient of $\tilde{\mathscr{U}}(\Sigma)$ for the $G_{\Xi}$-action.

In order to make computations, one still needs to determine the relations between the classes $l_{\rho}, \rho \in \Xi$. Proposition 2.5 implies that the diagram

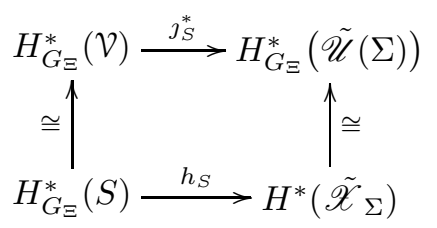

commutes. Since $h_{S}$ is surjective, $\jmath_{S}^{*}$ is surjective too, and it follows from the long exact sequence in equivariant cohomology for the pair $(\mathcal{V}, \tilde{\mathscr{U}}(\Sigma))$ that we have the short exact sequence

$$
0 \longrightarrow H_{G_{\Xi}}^{*}(\mathcal{V}, \tilde{\mathscr{U}}(\Sigma)) \stackrel{\imath_{S}^{*}}{\longrightarrow} H_{G_{\Xi}}^{*}(\mathcal{V}) \stackrel{\jmath_{S}^{*}}{\longrightarrow} H_{G_{\Xi}}^{*}(\tilde{\mathscr{U}}(\Sigma)) \longrightarrow 0
$$

and again $\imath_{S}^{*}$ and $\jmath_{S}^{*}$ are ring homomorphisms. Therefore

$$
\imath_{S}^{*} H_{G_{\Xi}}^{*}(\mathcal{V}, \tilde{\mathscr{U}}(\Sigma)) \subset H_{G_{\Xi}}^{*}(\mathcal{V})
$$

is an ideal, and our next task is to compute it.

Proposition 3.6. The Leray-Hirsch theorem does apply for the fibre bundle pair

$$
\left(E \times_{G_{\Xi}} V, E \times_{G_{\Xi}}\left(U(\Sigma) \times W_{0}^{\times}\right)\right) \hookrightarrow\left(E \times_{G_{\Xi}} \mathcal{V}, E \times_{G_{\Xi}} \tilde{\mathscr{U}}(\Sigma)\right)
$$

Proof. We have already described in the corollary 3.3 the generators for the cohomology of the fibre, and we must prove that they extend to classes in $H_{G_{\Xi}}^{*}(\mathcal{V}, \tilde{\mathscr{U}}(\Sigma))$. First of all, we should find the candidates for the cohomology extension. For any $\Sigma$-primitive family $\pi$, we define

$$
\mathcal{W}_{\pi}:=\bigoplus_{\beta \subset \pi} \mathbb{C}_{\beta} \otimes \mathcal{W}_{\beta}
$$

and we consider the projection

$$
\mathrm{pr}_{\pi}: \mathcal{V} \longrightarrow \mathcal{W}_{\pi}
$$

Denoting by $\operatorname{pr}_{S}: \mathcal{V} \rightarrow S$ the projection, we define the section

$$
s_{\pi}: \mathcal{V} \longrightarrow \operatorname{pr}_{S}^{*} \mathcal{W}_{\pi}, \quad v \longmapsto\left(v, \operatorname{pr}_{\pi}(v)\right)
$$

which is obviously $G_{\Xi}$-equivariant, and it vanishes nowhere on $\tilde{\mathscr{U}}(\Sigma) \subset \mathcal{V}$. Consequently, the restriction of the equivariant Euler class of $\operatorname{pr}_{S}^{*} \mathcal{W}_{\pi}$ to $\tilde{\mathscr{U}}(\Sigma)$ vanishes

$$
J_{S}^{*}\left(e_{G_{\Xi}}\left(\operatorname{pr}_{S}^{*} \mathcal{W}_{\pi}\right)\right)=0 \in H_{G_{\Xi}}^{*}(\tilde{\mathscr{U}}(\Sigma)) \text {. }
$$


The exactness of the sequence (3.8) implies now that

$$
e_{G_{\Xi}}\left(\operatorname{pr}_{S}^{*} \mathcal{W}_{\pi}\right) \in \imath_{S}^{*} H_{G_{\Xi}}^{*}(\mathcal{V}, \tilde{\mathscr{U}}(\Sigma)), \quad \text { for any primitive family } \pi \text {. }
$$

For every $j \in J$, we have the $G_{\Xi}$-equivariant section

$$
s_{j}: \mathcal{V} \rightarrow \operatorname{pr}_{S}^{*}\left(\underline{\mathbb{C}}_{j} \otimes \mathcal{V}_{j}\right), \quad v \longmapsto\left(v, v_{j}\right) .
$$

Again, this section vanishes nowhere on $\tilde{\mathscr{U}}(\Sigma)$, and therefore the restriction of the equivariant Euler class $e_{G_{\Xi}}\left(\operatorname{pr}_{S}^{*}\left(\underline{\mathbb{C}}_{j} \otimes \mathcal{V}_{j}\right)\right)$ to $\tilde{\mathscr{U}}(\Sigma)$ is zero. The exactness of $(3.8)$ implies again that

$$
e_{G \Xi}\left(\operatorname{pr}_{S}^{*}\left(\mathbb{C}_{j} \otimes \mathcal{V}_{j}\right)\right) \in \imath_{S}^{*} H_{G_{\Xi}}^{*}(\mathcal{V}, \tilde{\mathscr{U}}(\Sigma)), \quad \text { for any } j \in J .
$$

It is now clear, by corollary 3.3 , that the cohomology classes $\left(\imath_{S}^{*}\right)^{-1}\left(e_{G_{\Xi}}\left(\operatorname{pr}_{S}^{*} \mathcal{W}_{\pi}\right)\right)$ and $e_{G \Xi}\left(\operatorname{pr}_{S}^{*}\left(\underline{\mathbb{C}}_{j} \otimes \mathcal{V}_{j}\right)\right)$ define, in fact generate, a cohomology extension of the fibre bundle pair.

Corollary 3.7. The classes $\left(\imath_{S}^{*}\right)^{-1}\left(e_{G_{\Xi}}\left(\operatorname{pr}_{S}^{*} \mathcal{W}_{\pi}\right)\right)$, where $\pi$ ranges over the $\Sigma$-primitive families, and $\left(\imath_{S}^{*}\right)^{-1}\left(e_{G_{\Xi}}\left(\operatorname{pr}_{S}^{*}\left(\underline{\mathbb{C}}_{j} \otimes \mathcal{V}_{j}\right)\right)\right), j \in J$, generate $H_{G_{\Xi}}^{*}(\mathcal{V}, \tilde{\mathscr{U}}(\Sigma))$ as a $H_{G \Xi}^{*}(S)$-algebra.

Since the cohomology rings $H_{G_{\Xi}}^{*}(\mathcal{V})$ and $H_{G_{\Xi}}^{*}(S)$ are naturally isomorphic, we shall identify $e_{G_{\Xi}}\left(\operatorname{pr}_{S}^{*} \mathcal{W}_{\pi}\right)$ and $e_{G_{\Xi}}\left(\operatorname{pr}_{S}^{*}\left(\underline{\mathbb{C}}_{j} \otimes \mathcal{V}_{j}\right)\right)$ respectively with $e_{G_{\Xi}}\left(\mathcal{V}_{\pi}\right)$ and $e_{G \Xi}\left(\mathbb{C}_{j} \otimes \mathcal{V}_{j}\right)$. The explicit formulae for them are

$$
\begin{gathered}
e_{G_{\Xi}}\left(\mathcal{W}_{\pi}\right)=\prod_{b(\alpha) \subset \pi} e_{G_{\Xi}}\left(\underline{\mathbb{C}}_{\alpha} \otimes \mathcal{V}_{\alpha}\right)=\prod_{b(\alpha) \subset \pi}\left(\alpha^{r_{\alpha}}+c_{1}\left(\mathcal{V}_{\alpha}\right) \alpha^{r_{\alpha}-1}+\ldots+c_{r_{\alpha}}\left(\mathcal{V}_{\alpha}\right)\right) \\
e_{G \Xi}\left(\underline{\mathbb{C}}_{j} \otimes \mathcal{V}_{j}\right)=c_{1}\left(\mathcal{V}_{j}\right)+c_{\Xi}\left(f_{j}\right)=c_{1}\left(\mathcal{V}_{j}\right)+\hat{f}_{j} .
\end{gathered}
$$

Definition 3.8. We define the ideal of linear relations and the Stanley-Reisner ideal, both relative to $S$, respectively by

$$
\begin{aligned}
& \langle\mathcal{L} \mathcal{R}\rangle_{\Xi}:=H^{*}(S) \otimes\langle\mathrm{LR}\rangle_{\Xi}, \quad\langle\mathcal{L} \mathcal{R}\rangle_{\Sigma}:=H^{*}(S) \otimes\langle\mathrm{LR}\rangle_{\Sigma}, \\
& \left.\langle\mathcal{S R}\rangle_{\Xi}:=\left\langle e_{G_{\Xi}}\left(\mathcal{W}_{\pi}\right)\right| \pi \text { is a } \Sigma \text {-primitive family }\right\rangle \subset H_{G_{\Xi}}^{*}(S)=H_{G_{\Xi}}^{*} \otimes H^{*}(S), \\
& \left.\langle\mathcal{S R}\rangle_{\Sigma}:=\left\langle e_{G_{\Sigma}}\left(\mathcal{W}_{\pi}\right)\right| \pi \text { is a } \Sigma \text {-primitive family }\right\rangle \subset H_{G_{\Sigma}}^{*}(S)=H_{G_{\Sigma}}^{*} \otimes H^{*}(S) .
\end{aligned}
$$

By abuse of notation, in the next theorem, we shall still write $\langle\mathcal{S R}\rangle_{\Xi}$ and $\langle\mathcal{S R}\rangle_{\Sigma}$ for the inverse images of these ideals under the epimorphisms $H_{\left(\mathbb{C}^{*}\right)^{\Xi}} \otimes H^{*}(S) \rightarrow$ $H_{G_{\Xi}}^{*} \otimes H^{*}(S)$ and $H_{\left(\mathbb{C}^{*}\right)^{\Sigma(1)}}^{*} \otimes H^{*}(S) \rightarrow H_{G_{\Sigma}}^{*} \otimes H^{*}(S)$ respectively.

Now we are finally in position to compute the cohomology rings of $\tilde{\mathscr{X}}_{\Sigma}$ and $\mathscr{X}_{\Sigma}$.

Theorem 3.9. Let $\Sigma$ be a complete and simplicial fan, and consider $\mathcal{V} \rightarrow S$ a vector bundle.

(i) The cohomology ring of the family $\tilde{\mathscr{X}}_{\Sigma} \rightarrow S$ is

$$
\begin{aligned}
H^{*}\left(\tilde{\mathscr{X}}_{\Sigma} ; \mathbb{Q}\right) & \cong H_{G_{\Xi}}^{*}(S ; \mathbb{Q}) /\langle\mathcal{S R}\rangle_{\Xi}+\left\langle c_{1}\left(\mathcal{V}_{j}\right)+\hat{f}_{j}, j \in J\right\rangle \\
& \cong H^{*}(S ; \mathbb{Q})\left[f_{\rho} \mid \rho \in \Xi\right] /\langle\mathcal{L} \mathcal{R}\rangle_{\Xi}+\langle\mathcal{S R}\rangle_{\Xi}+\left\langle c_{1}\left(\mathcal{V}_{j}\right)+f_{j}, j \in J\right\rangle
\end{aligned}
$$

(ii) The cohomology ring of the family $\mathscr{X}_{\Sigma} \rightarrow S$ is

$$
H^{*}\left(\tilde{\mathscr{X}}_{\Sigma} ; \mathbb{Q}\right) \cong H_{G_{\Sigma}}^{*}(S ; \mathbb{Q}) /\langle\mathcal{S R}\rangle_{\Sigma} \cong H^{*}(S ; \mathbb{Q})\left[f_{\xi} \mid \xi \in \Sigma\right] /\langle\mathcal{L} \mathcal{R}\rangle_{\Sigma}+\langle\mathcal{S R}\rangle_{\Sigma} .
$$

If $\Sigma$ is regular, both isomorphisms hold with $\mathbb{Z}$-coefficients. 
Proof. (i) The key for the proof is the exact sequence (3.8): it implies that, as a ring,

$$
H^{*}\left(\tilde{\mathscr{X}}_{\Sigma}\right) \cong H_{G_{\Xi}}^{*}(\mathcal{V}) / \imath_{S}^{*} H_{G \Xi}^{*}(\mathcal{V}, \tilde{\mathscr{U}}(\Sigma))
$$

We should point out that since $\jmath_{S}^{*}$ is a ring homomorphism, $\imath_{S}^{*} H_{G_{\Xi}}^{*}(\mathcal{V}, \tilde{\mathscr{U}}(\Sigma)) \subset$ $H_{G_{\Xi}}^{*}(\mathcal{V})$ is an ideal, and the rôle of the previous discussion was to determine this ideal. Corollary 3.7 implies that

$$
\begin{aligned}
\imath_{S}^{*} H_{G_{\Xi}}^{*}(\mathcal{V}, \tilde{\mathscr{U}}(\Sigma)) & \cong \sum_{\pi \text { primitive }} H_{G_{\Xi}}^{*}(S) \cdot e_{G_{\Xi}}\left(\mathcal{W}_{\pi}\right)+\left\langle c_{1}\left(\mathcal{V}_{j}\right)+\hat{f}_{j}, j \in J\right\rangle \\
& =\langle\mathcal{S} \mathcal{R}\rangle_{\Xi}+\left\langle c_{1}\left(\mathcal{V}_{j}\right)+\hat{f}_{j}, j \in J\right\rangle .
\end{aligned}
$$

The first isomorphism in the statement of theorem becomes now clear. The second isomorphism is an immediate consequence of the fact that $H_{G_{\Xi}}^{*}(S)=H^{*}(S) \otimes H_{G_{\Xi}}^{*}$, and of the lemma 3.2 which states that $H_{G \Xi}^{*} \cong H_{\left(\mathbb{C}^{*}\right)^{\Xi}}^{*} /\langle\mathrm{LR}\rangle_{\Xi}$.

(ii) The structure of the cohomology ring of the family $\mathscr{X}_{\Sigma} \rightarrow S$ is obtained by replacing $\mathcal{V}$ with $\mathcal{W}_{\Sigma}$, or equivalently in the particular case when $\Sigma(1)=\Xi$.

What we should remark about this result is that the cohomology rings of the families $\tilde{\mathscr{X}}_{\Sigma}$ and $\mathscr{X}_{\Sigma}$ are not isomorphic in general, so that these families are really distinct despite the fact that both are $X_{\Sigma}$-fibrations over $S$. The reason for this phenomenon is that the isomorphism found in proposition 1.5 was not natural.

\section{A SPECial CASE}

Our primary motivation for studying families of toric varieties was their occurence in the context of the gauged sigma models. In this section we are going to put the phenomenon which occurs there in our present framework. In [5] and [9], the starting point was to consider a regular, projective fan $\Sigma \subset N_{\mathbb{R}}$ and its associated toric variety $X_{\Sigma}$. The first question one has to answer in order to compute enumerative invariants of $X_{\Sigma}$ is to describe the space of morphisms, with fixed degree, from a smooth and projective curve $C$ into $X_{\Sigma}$. It turns out that when this degree is sufficiently large, the compactification of this space of morphisms has the structure of a fibre bundle over the product of $b_{2}\left(X_{\Sigma}\right)$ copies of the Jacobian of $C$. In our language, it is a family of toric varieties parameterized by the base $S=\operatorname{Jac}(C)^{b_{2}\left(X_{\Sigma}\right)}$.

The fibres of this family are toric varieties which 'look very much the same' as the starting variety $X_{\Sigma}$. It is the meaning of this sentence which we intend to explain here. So let us consider a finite subset $\Xi \subset N$ having the properties enumerated in section 1. The diagram (1.2) provides us with the exact sequence

$$
0 \longrightarrow M \stackrel{a}{\longrightarrow} \mathbb{Z}^{\Xi} \stackrel{c}{\longrightarrow} A:=A_{\Xi} \longrightarrow 0,
$$

and therefore we can decompose

$$
\mathbb{Z}^{\Xi}=\bigoplus_{\xi \in \Xi} \mathbb{Z} f_{\xi}=\bigoplus_{\alpha \in A}\left(\bigoplus_{f_{\xi} \in c^{-1}(\alpha)} \mathbb{Z} f_{\xi}\right)=: \bigoplus_{\alpha \in A} L_{\alpha}
$$

Each of the $L_{\alpha}$ 's are lattices, since they are torsion free $\mathbb{Z}$-modules. Let us choose arbitrary, strictly positive integers $\left\{n_{\alpha}\right\}_{\alpha \in A}$ and define

$$
L^{\prime}:=\bigoplus_{\alpha \in A} L_{\alpha}^{\oplus n_{\alpha}}
$$


This amounts saying that for every $\alpha \in A$ we reproduce a number of $n_{\alpha}$ times each vector $f_{\xi} \in c^{-1}(\alpha)$. For fixed $\alpha \in A$ and $f_{\xi} \in c^{-1}(\alpha)$, we denote by $\left\{f_{\xi 1}, \ldots, f_{\xi n_{\alpha}}\right\}$ these vectors, so we can write

$$
L^{\prime}=\bigoplus_{\alpha \in A}\left(\bigoplus_{f_{\xi} \in c^{-1}(\alpha)}\left(\mathbb{Z} f_{\xi 1} \oplus \ldots \oplus \mathbb{Z} f_{\xi n_{\alpha}}\right)\right) .
$$

It is now clear that there is a natural surjective homomorphism

$$
c^{\prime}: L^{\prime} \longrightarrow A, \quad f_{\xi} \longmapsto c\left(f_{\xi}\right) \in A .
$$

Let us denote by $M^{\prime}:=\operatorname{ker}\left(c^{\prime}\right)$ and by $a^{\prime}: M^{\prime} \rightarrow L^{\prime}$ the inclusion. We notice that $M^{\prime}$ is a lattice, since without torsion, whose rank is

$$
\operatorname{rk}\left(M^{\prime}\right)=\operatorname{rk}(M)+\sum_{\alpha \in A}\left(n_{\alpha}-1\right) \operatorname{rk}\left(L_{\alpha}\right) .
$$

The lattice $L^{\prime}$ 'looks very much like' $\mathbb{Z}^{\Xi}$ in the following sense: for every $\alpha \in A$ and $f_{\xi} \in c^{-1}(\alpha)$, and for any choice of $\bullet_{\xi} \in\left\{1, \ldots, n_{\alpha}\right\}$, we find the monomorphism

$$
j .: \mathbb{Z}^{\Xi} \longrightarrow L^{\prime}, \quad f_{\xi} \longmapsto f_{\xi \cdot \xi} .
$$

There is also a canonical epimorphism

$$
+: L^{\prime}=\bigoplus_{\alpha \in A} L_{\alpha}^{n_{\alpha}} \longrightarrow \bigoplus_{\alpha \in A} L_{\alpha}=\mathbb{Z}^{\Xi}
$$

induced by the natural additions $+_{\alpha}: L_{\alpha}^{n_{\alpha}} \rightarrow L_{\alpha}, \alpha \in A$. Let us notice now that for all choices of $j$. the diagram

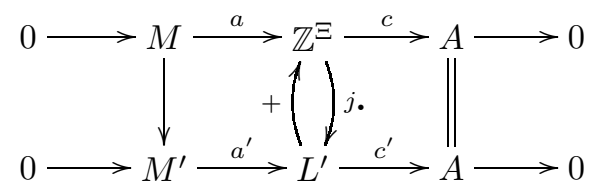

commutes. By dualizing, we get for $N^{\prime}:=\operatorname{Hom}_{\mathbb{Z}}\left(M^{\prime}, \mathbb{Z}\right)$ the commutative diagram

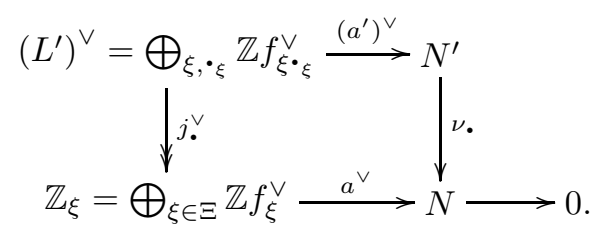

and observe that the homomorphism $\nu$. is surjective. We define now the set $\Xi^{\prime}:=$ $\left\{\left(a^{\prime}\right)^{\vee}\left(f_{\xi \bullet}^{\vee}\right)\right\} \subset N^{\prime}$, where $f_{\xi \bullet}^{\vee}$ stays for the canonical generator of $\operatorname{Hom}_{\mathbb{Z}}\left(\mathbb{Z} f_{\xi}, \mathbb{Z}\right)$. Our goal is to prove the following

Lemma 4.1. The set $\Xi^{\prime} \subset N^{\prime}$ fulfills the conditions (i)-(iii) and $L^{\prime} \cong \mathbb{Z}^{\Xi^{\prime}}$.

Proof. We claim that $0 \notin \Xi^{\prime}$ : otherwise we find an $f_{\xi_{0}} \cdot \xi_{0}$ having the property that all vectors in $M^{\prime}$ have the corresponding entry zero in $L^{\prime}$. We choose now an inclusion $j$. such that $f_{\xi_{0}} \mapsto f_{\xi_{0}}{ }_{\xi_{0}}$. Then it follows from the diagram above that

$$
\xi_{0}=a^{\vee}\left(f_{\xi_{0}}^{\vee}\right)=a^{\vee}\left(j \vee\left(f_{\xi_{0} \cdot \xi_{0}}^{\vee}\right)\right)=0,
$$

and this is a contradiction.

We are going to check now the second property, that each $\xi^{\prime}=\left(a^{\prime}\right)^{\vee}\left(f_{\xi_{\xi}}\right)$ is the generator over $\mathbb{Z}_{\geq 0}$ of the semi-group $\mathbb{R}_{\geq 0} \xi^{\prime} \cap N^{\prime}$. We chose an appropriate 
inclusion $j$. as above. Then we know that $\xi=\nu \cdot\left(\xi^{\prime}\right)=a^{\vee}\left(f_{\xi}^{\vee}\right)$ is the generator of the semi-group $\mathbb{Z}_{\geq 0} \xi \cap N$ and therefore $\xi^{\prime}$ has the same property in $N^{\prime}$.

It remains to check that $\sum_{\xi^{\prime} \in \Xi^{\prime}} \mathbb{R}_{\geq 0} \xi^{\prime}=N_{\mathbb{R}}^{\prime}$. The left hand side is a convex cone in $N_{\mathbb{R}}^{\prime}$, and the condition is equivalent asking that there is no hyperplane in $N_{\mathbb{R}}^{\prime}$ which leaves this cone on one of its sides. In other words, it is enough checking that there is no $m^{\prime} \in M^{\prime}$ such that $\left\langle m^{\prime}, \xi^{\prime}\right\rangle \geq 0$, for all $\xi^{\prime} \in \Xi^{\prime}$. Let us assume the contrary that such an $m^{\prime} \in M^{\prime} \backslash\{0\}$ does exist. Then $\left(c \circ+\circ a^{\prime}\right)\left(m^{\prime}\right)=0 \in A$, and consequently $\left(+\circ a^{\prime}\right)\left(m^{\prime}\right)=a(m)$ for some $m \in M$. This $m$ is certainly not zero, because $a\left(m^{\prime}\right)$ has posivive entries and has at least one strictly positive entry. Then we have found an element $m \in M \backslash\{0\}$ which leaves all the $\xi \in \Xi$ on one of its sides. This is a contadiction with the fact that $\Xi \subset N$ does obey (iii).

We must still check a tacit assumption, namely that the $\xi^{\prime}$ 's are indeed pairwise distinct. Let us assume the contrary, that there exist $f_{\xi_{1} \cdot{ }_{1}}$ and $f_{\xi_{2} \cdot{ }_{2}}$ which determine the same element in $N^{\prime}$. This means that for any $m^{\prime} \in M^{\prime}$, the element $a^{\prime}\left(m^{\prime}\right) \in L^{\prime}$ has the $\xi_{1} \bullet_{1}$ and the $\xi_{2} \bullet_{2}$ coordinates equal. But if follows from the definition of $M^{\prime}$ that

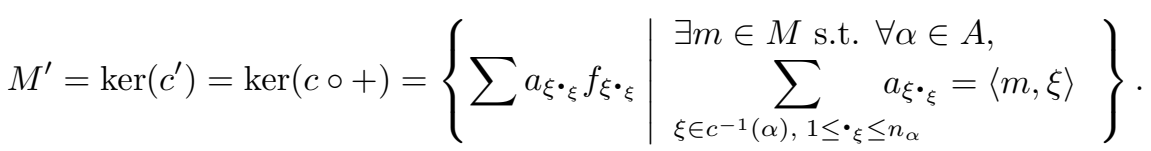

We distinguish between two possibilities: the first one is when $\xi_{1}=\xi_{2}=\xi$. We can choose in this case the vector whose $\xi_{1}$ and $\xi_{2}$ entries are 1 and -1 respectively, and zero in rest. It clearly belongs to $\operatorname{ker}\left(c^{\prime}\right)=M^{\prime}$, but however the entries of interest are different. The second case is when $\xi_{1} \neq \xi_{2}$ : then we can choose a monomorphism $j \cdot: \mathbb{Z}^{\Sigma(1)} \rightarrow L^{\prime}$ which sends $f_{\xi_{1}} \mapsto f_{\xi_{1} \bullet_{1}}$ and $f_{\xi_{2}} \mapsto f_{\xi_{2} \cdot \bullet_{2}}$. We deduce then that $\xi_{1}=a^{\vee}\left(f_{\xi_{1}}\right)=a^{\vee}\left(f_{\xi_{2}}\right)=\xi_{2}$, which is again a contradiction. Therefore the vectors $\xi^{\prime} \in N^{\prime}$ defined above are pairwise distinct.

The last property in the lemma follows now from the definions of $N^{\prime}$ and $\Xi^{\prime}$.

We can finally explain the situation which appears in [5, 9]: $\Sigma \in N_{\mathbb{R}}$ is a projective and regular fan, and $\Xi=\Sigma(1)$. One defines a set $\Xi^{\prime} \subset N^{\prime}$ as above, and the space of morphisms with fixed degree from a curve $C$ into $X_{\Sigma}$ turns out to be a toric fibration over $\operatorname{Jac}(C)^{b_{2}\left(X_{\Sigma}\right)}$. The fibres are toric varieties associated to the fan $\Sigma^{\prime} \subset N_{\mathbb{R}}^{\prime}$ whose primitive families are

$$
\left\{\left(a^{\prime}\right)^{\vee}\left(f_{\xi_{\xi}}^{\vee}\right) \mid \xi \in \pi, \cdot \xi \in\left\{1, \ldots, n_{c\left(f_{\xi}\right)}\right\}\right\}
$$

with $\pi \subset \Xi$ a $\Sigma$-primitive family. The maximal cones of this fan can be described as follows: for each cone $\sigma \in \Sigma_{\max }$, choose $k_{\xi} \in\left\{1, \ldots, n_{c\left(f_{\xi}\right)}\right\}$, for all $\xi \notin \sigma(1)$; define then $\sigma^{\prime} \in \Sigma_{\max }^{\prime}$ to be the cone whose 1-skeleton is

$$
\sigma^{\prime}(1)=\left\{\begin{array}{l|l}
\left(a^{\prime}\right)^{\vee}\left(f_{\xi_{\xi}}\right) & \begin{array}{l}
\bullet_{\xi} \in\left\{1, \ldots, n_{c\left(f_{\xi}\right)}\right\}, \quad \forall \xi \in \sigma(1) \\
\bullet_{\xi} \in\left\{1, \ldots, n_{c\left(f_{\xi}\right)}\right\} \backslash\left\{k_{\xi}\right\}, \quad \forall \xi \notin \sigma(1)
\end{array}
\end{array}\right\} .
$$

Lemma 4.2. The fan $\Sigma^{\prime}$ is projective and regular.

Proof. Since $\Sigma$ is regular, $X_{\Sigma}$ is isomorphic to the geometric quotient $U(\Sigma) / G$, and $U(\Sigma) \rightarrow X_{\Sigma}$ is a principal $G$-bundle, where $G=\operatorname{Hom}_{\mathbb{Z}}\left(A, \mathbb{C}^{*}\right)$. Moreover, since $\Sigma$ is projective, there exists an ample class $\chi \in A=A^{1}\left(X_{\Sigma}\right)$, and $X_{\Sigma}$ can also be described as the geometric invariant quotient $\mathbb{C}^{\Xi} / / G$ for the linearization

$$
G \times\left(\mathbb{C}^{\Xi} \times \mathbb{C}\right) \longrightarrow \mathbb{C}^{\Xi} \times \mathbb{C}, \quad g \times(z, \varepsilon)=(g \times z, \chi(g) \varepsilon) .
$$


Since $\sum_{\xi^{\prime} \in \Xi^{\prime}} \mathbb{R}_{\geq 0} \xi^{\prime}=N^{\prime}$, we deduce that $\Sigma^{\prime}$ is complete. Cox's result says that $X_{\Sigma^{\prime}}$ is (canonically) isomorphic to the categorical quotient $U^{\prime}\left(\Sigma^{\prime}\right) / G$, and

$$
U^{\prime}\left(\Sigma^{\prime}\right)=\mathbb{C}^{\Xi^{\prime}} \backslash Z_{\Sigma^{\prime}}=\bigcup_{\sigma^{\prime} \in \Sigma_{\max }^{\prime}} U_{\sigma^{\prime}}^{\prime} \quad \text { with } \quad U_{\sigma^{\prime}}^{\prime}=\left\{\prod_{\xi \notin \sigma(1)} Z_{\xi k_{\xi}} \neq 0 .\right\} .
$$

We linearize the $G$-action on $\mathbb{C}^{\Xi^{\prime}}$ in the trivial line bundle, using the character $\chi$, and we want to prove that

$$
U^{\prime}\left(\Sigma^{\prime}\right) \subseteq\left(\mathbb{C}^{\Xi^{\prime}}\right)^{\mathrm{ss}(\chi)}
$$

We will deduce then that $X_{\Sigma^{\prime}}$ is quasi-projective and complete, and is consequently projective. A posteriori we find that the inclusion above is in fact an equality.

Let us consider a maximal cone $\sigma^{\prime}$ of $\Sigma^{\prime}$, and choose an inclusion $j .: \mathbb{C}^{\Xi} \rightarrow \mathbb{C}^{\Xi^{\prime}}$ having the property that $Z_{\xi} \mapsto Z_{\xi k_{\xi}}$, for all $\xi \notin \sigma(1)$. We denote by $p .: \mathbb{C}^{\Xi^{\prime}} \rightarrow \mathbb{C}^{\Xi}$ the corresponding projection, and we notice that both $j$. and $p$. are $G$-equivariant. Moreover, equality (4.2) implies that $U_{\sigma}=p \cdot\left(U_{\sigma^{\prime}}^{\prime}\right)$.

If $z^{\prime} \in U^{\prime}\left(\Sigma^{\prime}\right)$, there is a maximal cone $\sigma^{\prime} \in \Sigma^{\prime}$ such that $z^{\prime} \in U_{\sigma^{\prime}}^{\prime}$. Then $z=p \cdot\left(z^{\prime}\right) \in U_{\sigma} \subset U(\Sigma)$, and we find an integer $\kappa \geq 1$ and $s \in \mathbb{C}\left[\mathbb{C}^{\Xi}\right]^{\chi^{\kappa}}$ such that $s(z) \neq 0$. Then $(p .)^{*} s$ is a $\chi^{\kappa}$-invariant section on $\mathbb{C}^{\Xi^{\prime}}$ which does not vanish at $z^{\prime}$. The inclusion (4.3) is proved now.

We must still show that $\Sigma$ is regular. The first thing to notice is that $U^{\prime}\left(\Sigma^{\prime}\right) \rightarrow$ $X_{\Sigma^{\prime}}$ is actually a geometric quotient. It is enough to check that all $G$-orbits are closed in $U_{\sigma^{\prime}}^{\prime}$, for every $\sigma^{\prime} \in \Sigma_{\max }^{\prime}$. This follows from the following facts: first, since the quotient $U(\Sigma) \rightarrow X_{\Sigma}$ is geometric, all the $G$-orbits are closed in $U_{\sigma}$, for all $\sigma \in \Sigma_{\max }$; secondly, the image of the inclusion $j .: \mathbb{C}^{\Xi} \rightarrow \mathbb{C}^{\Xi^{\prime}}$ defined above has closed image and $U_{\sigma}=j_{.}^{-1}\left(U_{\sigma^{\prime}}^{\prime}\right)$. Then its restiction to $U_{\sigma}$ still has closed image in $U_{\sigma^{\prime}}^{\prime}$ and is $G$-equivariant. Therefore the $G$-orbits in $U_{\sigma^{\prime}}^{\prime}$ are closed, hence the quotient $U^{\prime}\left(\Sigma^{\prime}\right) \rightarrow X_{\Sigma^{\prime}}$ is geometric, as stated. Using again Cox's theorem [1, theorem 2.1], we deduce that $\Sigma^{\prime}$ is simplicial.

Moreover, $\Sigma$ being regular, $G$ acts freely on each affine piece $U_{\sigma}$. Using the inclusion $j$., we deduce that $G$ acts freely on $U_{\sigma^{\prime}}^{\prime}$ too, so that $X_{\Sigma^{\prime}}$ is smooth. We conclude now from [3, page 29] that $\Sigma^{\prime}$ is regular.

The statement, at the beginning of this section, that the toric variety $X_{\Sigma^{\prime}}$ 'looks the same' as $X_{\Sigma}$ means, after this discussion, that through each point of $X_{\Sigma^{\prime}}$ passes a $\sum_{\alpha \in A}\left(n_{\alpha}-1\right) \operatorname{rk}\left(L_{\alpha}\right)$-dimensional algebraic family of embedded copies of $X_{\Sigma}$.

The simplest exemple is when $X_{\Sigma}=\mathbb{P}^{r-1}$ and $X_{\Sigma^{\prime}}=\mathbb{P}^{n r-1}$. In this case through each point of $\mathbb{P}^{n r-1}$ passes a family of linearly embedded copies of $\mathbb{P}^{r-1}$ 's, parameterized by $\mathbb{P}^{(n-1) r}$.

\section{APPENDIX}

For proving proposition 4.6, D. Cox makes use in [1, page 43] of the following

Lemma Let $\Sigma \subset N_{\mathbb{R}}$ be a complete fan and consider $m \in M=N^{\vee}$ such that the corresponding hyperplane $\mathcal{H}_{m}:=\{\langle m, \cdot\rangle=0\} \subset N_{\mathbb{R}}$ has the properties:

(i) there exists a unique $\xi_{0} \in \Sigma(1)$ with $\left\langle m, \xi_{0}\right\rangle>0$,

(ii) there is a cone $\sigma_{0} \in \Sigma$ included in $\mathcal{H}_{m}$.

Then the cone $\mathbb{R}_{\geq 0} \xi_{0}+\sigma_{0}$ belongs to $\Sigma$. 
In loc. cit. the lemma is stated for $\Sigma$ complete and simplicial and in this case the proof is rather clear. What we are going to show here is that the lemma still holds in the more general setting when $\Sigma$ is complete.

Proof. Let us consider the star of $\sigma_{0}$, which is by definition the set

$$
\operatorname{Star}\left(\sigma_{0}\right):=\left\{\sigma \in \Sigma \mid \sigma_{0} \subset \sigma\right\}
$$

Since $\Sigma$ is complete,

$$
\Sigma_{0}:=\left\{\sigma /\left\langle\sigma_{0}\right\rangle \mid \sigma \in \operatorname{Star}\left(\sigma_{0}\right)\right\}
$$

is a complete fan in $N_{\mathbb{R}} /\left\langle\sigma_{0}\right\rangle$. We have used the symbol $\left\langle\sigma_{0}\right\rangle$ to denote the vector space generated by the cone $\sigma_{0}$. Since $\sigma_{0} \in \operatorname{Ker}(m), m$ descends to a linear functional $\hat{m}$ on the quotient

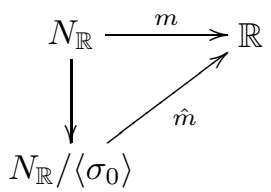

and the correponding hyperplane $\mathcal{H}_{\hat{m}}$ breaks $N_{\mathbb{R}} /\left\langle\sigma_{0}\right\rangle$ into two half-spaces. As $\Sigma_{0}$ is complete, it must exist an element of $\Sigma_{0}(1)$ situated on the $\left.\{\langle\hat{m}, \cdot\rangle\rangle 0\right\}$-side of $\mathcal{H}_{\hat{m}}$. But we know from the hypothesis that the only element of $\Sigma(1)$ which is on the $\{\langle m, \cdot\rangle>0\}$-side of $\mathcal{H}_{m}$ is $\xi_{0}$. We deduce that the cone generated by $\hat{\xi}_{0}:=\operatorname{proj}_{N_{\mathbb{R}} /\left\langle\sigma_{0}\right\rangle}\left(\xi_{0}\right)$ is necessarily an element of $\Sigma_{0}(1)$. This means that there is a cone $\sigma^{\prime} \in \operatorname{Star}\left(\sigma_{0}\right) \subset \Sigma$ such that $\sigma^{\prime} /\left\langle\sigma_{0}\right\rangle=\mathbb{R}_{\geq 0} \hat{\xi}_{0}$, and consequently there is a 1-dimensional cone of $\sigma^{\prime}$ which projects onto $\mathbb{R}_{\geq 0} \hat{\xi}_{0}$. But, we use the unicity of $\xi_{0}$ again, and we deduce that in fact $\mathbb{R}_{\geq 0} \xi_{0}$ must be 1-dimensional cone of $\sigma^{\prime}$.

For the moment we have found a cone $\sigma^{\prime}$ of $\Sigma$ with the properties

$$
\mathbb{R}_{\geq 0} \xi_{0}+\sigma_{0} \subset \sigma^{\prime} \quad\left(\xi_{0} \in \sigma^{\prime}, \sigma_{0} \subset \sigma^{\prime}\right)
$$

and

$$
\sigma^{\prime} \subset \mathbb{R}_{\geq 0} \xi_{0}+\left\langle\sigma_{0}\right\rangle \quad\left(\sigma^{\prime} /\left\langle\sigma_{0}\right\rangle=\mathbb{R}_{\geq 0} \hat{\xi}_{0}\right) .
$$

We deduce that $\sigma^{\prime}$ is included in the $\{\langle m, \cdot\rangle \geq 0\}$-side of $\mathcal{H}_{m}$, and consequently $\sigma^{\prime} \cap \mathcal{H}_{m}$ is a face of $\sigma^{\prime}$; in particular, $\sigma^{\prime} \cap \mathcal{H}_{m}$ is an element of $\Sigma$. Using the previous two properties of $\sigma^{\prime}$, we find that

$$
\sigma_{0} \subset \sigma^{\prime} \cap \mathcal{H}_{m} \subset\left\langle\sigma_{0}\right\rangle
$$

As both $\sigma_{0}$ and $\sigma^{\prime} \cap \mathcal{H}_{m}$ are elements of $\Sigma$, one of the defining properties of a fan implies that $\sigma_{0} \cap\left(\sigma^{\prime} \cap \mathcal{H}_{m}\right)=\sigma_{0}$ is a face of $\sigma^{\prime} \cap \mathcal{H}_{m}$. This is possible only if $\sigma^{\prime} \cap \mathcal{H}_{m}=\sigma_{0}$ because $\sigma^{\prime} \cap \mathcal{H}_{m} \subset\left\langle\sigma_{0}\right\rangle$. We conclude now that $\sigma^{\prime}(1)=\mathbb{R}_{\geq 0} \xi \cup \sigma(1)$ and consequently $\mathbb{R}_{\geq 0} \xi_{0}+\sigma_{0}=\sigma^{\prime}$ is an element of $\Sigma$.

\section{REFERENCES}

[1] D. Cox: The homogeneous coordinate ring of a toric variety, J. Algebr. Geom. 4 (1995), $17-50$

[2] V.I. Danilov: The geometry of toric varieties, Russian Math. Surveys 33 (1978), 97-154

[3] W. Fulton: Introduction to toric varieties, Annals of Mathematics Studies no. 131, Princeton University Press, 1993

[4] W. Fulton: Intersection Theory, Springer-Verlag Berlin Heidelberg New York Tokyo 1984

[5] M. Halic: Higher genus curves on toric varieties, preprint AG/0101100

[6] D. Husemoller: Fibre Bundles $2^{\text {nd }}$ ed. Springer-Verlag 1975 
[7] H. Kraft: G-vector bundles and the linearization problem, Canadian Math. Soc. Conference Proceedings 10 (1989), 111-123

[8] T. Oda, H. Park: Linear Gale transforms and the Gelfand-Kapranov-Zelevinskij decompositions, Tohôku Math. J. 43 (1991), 375-399

[9] Ch. Okonek, A. Teleman: Gauge theoretical Gromov-Witten invariants for toric varieties. Comparing virtual fundamental classes, preprint

[10] E. Spanier: Algebraic Topology, McGraw-Hill Inc. 1966

InStitut Für Mathematik, Universität ZÜRICH, WinterthURERSTRASSE 190, CH-8057 ZÜRICH, SWITZERLAND

E-mail address: halic@math.unizh.ch 